\title{
THE DUST CONTENT AND OPACITY OF ACTIVELY STAR-FORMING GALAXIES ${ }^{1}$
}

\author{
Daniela Calzetti \\ Space Telescope Science Institute, 3700 San Martin Drive, Baltimore, MD 21218; calzetti@stsci.edu \\ LeE ARMUS \\ SIRTF Science Center/Caltech, Pasadena, CA 91125; lee@ipac.caltech.edu \\ RALPH C. BoHLIN AND ANNE L. KinNeY \\ Space Telescope Science Institute, MD 21218; bohlin@stsci.edu; kinney@stsci.edu \\ JAN KOORNNEEF \\ Kapteyn Astronomical Institute, University of Groningen, Groningen, 9700 AV, Netherlands; J.Koornneef@astro.rug.nl \\ AND \\ THAISA STORCHI-BERgMANN \\ Instituto de Fisica, Universidade Federal Rio Grande do Sul, Porto Alegre, RS 91501-970, Brazil; thaisa@if.ufrgs.br \\ Received 1999 July 19; accepted 1999 November 30
}

\begin{abstract}
We present far-infrared (FIR) photometry at 150 and $205 \mu \mathrm{m}$ of eight low-redshift starburst galaxies obtained with the Infrared Space Observatory (ISO) ISOPHOT. Five of the eight galaxies are detected in both wave bands, and these data are used, in conjunction with IRAS archival photometry, to model the dust emission at $\lambda \gtrsim 40 \mu \mathrm{m}$. The FIR spectral energy distributions (SEDs) are best fitted by a combination of two modified Planck functions, with $T \sim 40-55 \mathrm{~K}$ (warm dust) and $T \sim 20-23 \mathrm{~K}$ (cool dust) and with a dust emissivity index $\epsilon=2$. The cool dust can be a major contributor to the FIR emission of starburst galaxies, representing up to $60 \%$ of the total flux. This component is heated not only by the general interstellar radiation field, but also by the starburst itself. The cool dust mass is up to $\sim 150$ times larger than the warm dust mass, bringing the gas-to-dust ratios of the starbursts in our sample close to Milky Way values, once rescaled for the appropriate metallicity. The ratio between the total dust FIR emission in the range 1-1000 $\mu \mathrm{m}$ and the IRAS FIR emission in the range $40-120 \mu \mathrm{m}$ is $\sim 1.75$, with small variations from galaxy to galaxy. This ratio is about $40 \%$ larger than previously inferred from data at millimeter wavelengths. Although the galaxies in our sample are generally classified as "UV bright," for four of them the UV energy emerging shortward of $0.2 \mu \mathrm{m}$ is less than $15 \%$ of the FIR energy. On average, about $30 \%$ of the bolometric flux is coming out in the UV-to-near-IR wavelength range; the rest is emitted in the FIR. Energy balance calculations show that the FIR emission predicted by the dust reddening of the UV-to-near-IR stellar emission is within a factor of $\sim 2$ of the observed value in individual galaxies and within $20 \%$ when averaged over a large sample. If our sample of local starbursts is representative of high-redshift $(z \gtrsim 1)$, UV-bright, star-forming galaxies, these galaxies' FIR emission will be generally undetected in submillimeter surveys, unless (1) their bolometric luminosity is comparable to or larger than that of ultraluminous FIR galaxies and (2) their FIR SED contains a cool dust component.
\end{abstract}

Subject headings: galaxies: starburst — infrared: galaxies — infrared: ISM: continuum ISM: dust, extinction

\section{INTRODUCTION}

The far-infrared (FIR) spectral energy distributions (SEDs) of galaxies provide insight into the energetic processes taking place in the sources. Emission beyond a few microns from quiescent or star-forming galaxies is dominated by dust reradiating the stellar energy absorbed at UV-optical wavelengths. Thus, the description of the radiative processes in the FIR regime needs to account for both the dust composition and the nature of the heating sources.

Although dust emission is a complex process involving a continuum of values for the properties of the dust grains, a simple two- (or three-) component dust model has proven successful at describing the basic characteristics of the UVoptical extinction and the FIR emission of the dust in our

\footnotetext{
${ }^{1}$ Based on observations with ISO, an ESA project with instruments funded by ESA member states (especially the PI countries: France, Germany, the Netherlands, and the United Kingdom) with the participation of ISAS and NASA.
}

own and similar galaxies (Andriesse 1978; Draine \& Anderson 1985; Cox, Krügel, \& Mezger 1986; Mathis \& Whiffen 1989; Puget \& Léger 1989; Desért, Boulanger, \& Puget 1990; Helou, Ryter, \& Soifer 1991; Rowan-Robinson 1992). One component, sometimes split into two (Desért et al. 1990), consists of very small grains and large molecules (size $\lesssim 100 \AA$ ) which account for characteristics of the mid-IR emission at $\lambda \lesssim 40-50 \mu \mathrm{m}$. This component is heated by the single-photon absorption process to temperatures of a few hundred Kelvin, up to $\sim 1000 \mathrm{~K}$, and is not in thermal equilibrium with its environment (Sellgren 1984; Draine \& Anderson 1985). The second component is formed by "large" grains (size $>100 \AA$ ) in thermal equilibrium with their environment. These large dust grains account for practically all the emission longward of 80-100 $\mu \mathrm{m}$ in galaxies. Their FIR emission can be modeled as the superposition of multiple modified Planck functions covering a range of temperatures and modulated by the grains' emissivity $e(v) \propto v^{\epsilon}$, with $1 \leq \epsilon \leq 2$ (e.g., Seki \& Yamamoto 
1980; Mezger, Mathis, \& Panagia 1982; Hildebrand 1983; Andriesse 1974; Bianchi, Davies, \& Alton 1999).

The color temperatures of the large dust grains depend on both the energy density per unit volume and the hardness of the stellar radiation field. As a first approximation, the galaxy FIR emission longward of $40-50 \mu \mathrm{m}$ has been ascribed to dust heated by massive, ionizing stars (warm dust) if the color temperature is of the order of $30-70 \mathrm{~K}$ and to dust heated by nonionizing stars (cool dust) if the temperatures are $\lesssim 15-30 \mathrm{~K}$ (e.g., Helou 1986; Chini, Krügel, \& Kreysa 1986b; Lonsdale-Persson \& Helou 1987; RowanRobinson \& Crawford 1989; Rowan-Robinson \& Efstathiou 1993). The radiation field created by the diffuse nonionizing population of a galaxy is generally referred to as the interstellar radiation field (ISRF) (e.g., Boulanger et al. 1988). Massive stars can also heat the dust to color temperatures $T \gtrsim 70 \mathrm{~K}$; this hot dust contributes, together with the small, nonequilibrium grains, mainly to the mid-IR emission shortward of $\sim 40 \mu \mathrm{m}$, typically with decreasing filling factor for increasing temperature (e.g., Natta \& Panagia 1976). The intensity of the dust emission at $\lambda \sim 10$ $\mu \mathrm{m}$ has been observed to decrease for increasing star formation activity in a galaxy (Helou 1986); this has been interpreted as the destruction of large molecules/very small grains by hard radiation from massive stars (Boulanger et al. 1988). Throughout this paper, "thermal FIR emission" refers to the emission in the wavelength range 1-1000 $\mu \mathrm{m}$ from large dust grains with equilibrium temperatures $T \lesssim$ 60-70 K. "Total FIR emission" defines the integrated dust emission from both small and large grains, also in the range $1-1000 \mu \mathrm{m}$.

The largest database of FIR data of galaxies available to date is from the IRAS survey (e.g., Soifer et al. 1989). Because the wavelength coverage is $8-120 \mu \mathrm{m}, I R A S$ observations alone are not adequate for characterizing the emission from dust cooler than $T \approx 30 \mathrm{~K}$. However, cool dust has been shown to be a major contributor to the total dust mass content of a galaxy (e.g., Kwan \& Xie 1992; Xu \& Helou 1986) and to contribute to the opacity budget. In relatively recent years, observations at millimeter and submillimeter wavelengths have been used to complement IRAS data, to address the issue of the cool dust content of galaxies (e.g., Chini et al. 1986a; Kwan \& Xie 1992; Roche \& Chandler 1993; Sievers et al. 1994; Rigopoulou, Lawrence, \& Rowan-Robinson 1996; Andreani \& Franceschini 1996, to quote a few). One of the drawbacks of millimeter observations is the potential presence of contamination from thermal and nonthermal radio emission (e.g., Roche \& Chandler 1993). Less sensitive to this problem, but still effective at characterizing dust emission down to temperatures of $\lesssim 15 \mathrm{~K}$, are observations obtained with the Infrared Space Observatory (ISO) satellite, which covered the FIR wavelength range up to $\sim 240 \mu \mathrm{m}$. ISO data have shown that the FIR emission of late-type galaxies receives a substantial contribution from cool dust $(T \sim 10-20 \mathrm{~K}$; Krügel et al. 1998; Haas et al. 1998; Alton et al. 1998; Domingue et al. 1999), increasing by a factor of $2-3$, up to 10 , the dust content of such systems relative to previous estimates, often based on IRAS data alone; the cool dust component is extended beyond the optical disk and produces gas-to-dust ratios comparable to average Milky Way values (Alton et al. 1998; Davies et al. 1999). Cool dust is also present in elliptical galaxies (Haas 1998), complementing the IRAS detections of relatively warm dust,
$T \sim 25-30 \mathrm{~K}$, in these objects (e.g., Goudfrooij \& de Jong 1995). Conversely, the $I S O$ emission of luminous and ultraluminous FIR galaxies, like Arp 244, NGC 6240, Arp 220, and M82, are fitted by a modified Planck function with color temperature $\sim 30-50 \mathrm{~K}$ for emissivity index $\epsilon=1$ (Klaas et al. 1997; Colbert et al. 1999).

Our investigation concentrates on actively star-forming galaxies, because of the key role they play in interpreting high-redshift galaxies. Some of the observational characteristics of high-redshift, $z \sim 3$ and $z \sim 4$, Lyman-break galaxies (Steidel et al. 1996, 1999; Giavalisco et al. 1996, 2000) are similar to those of the central regions of local starburst galaxies. Such characteristics include the star formation rates per unit area, the shape of the stellar continuum and the intensity and shape of the absorption features in the rest-frame UV spectra, and the ionizing/UV-continuum photon ratios (Steidel et al. 1996; Pettini et al. 1998; Meurer, Heckman, \& Calzetti 1999). Still open is the issue of the dust opacity in these UV-bright, star-forming systems. A new challenge to the common wisdom that there is little dust opacity in the young universe has come from the discovery of FIR-bright galaxies at intermediate/high redshift with the submillimeter common-user bolometer array (SCUBA) (Hughes et al. 1998; Eales et al. 1998; Blain et al. 1999; Lilly et al. 1999a; Barger et al. 1999). The necessity of linking the two populations of high-redshift galaxies, UV bright and FIR bright, has clearly brought new urgency to the problem of understanding the opacity and dust emission characteristics of star-forming galaxies at all redshifts.

This paper presents ISO photometric observations of eight nearby star-forming galaxies in the wavelength range $120-240 \mu \mathrm{m}$, in order to study the galaxies' dust content in a wavelength range which is beyond the limits of IRAS. For five of the galaxies, the balance between the UV-opticalnear-IR stellar energy absorbed by the dust and the FIR energy emitted by dust is investigated in detail. The results from our small sample will be placed in the more general context of dust opacity in star-forming galaxies at low and high redshift.

\section{SAMPLE SELECTION, OBSERVATIONAL STRATEGY, AND DATA REDUCTION}

The original sample of 40 star-forming galaxies from which our ISO sources were selected was derived from the IUE Atlas by Kinney et al. (1993), with the following characteristics: the galaxies are UV bright and have star formation rates (SFRs) of a few up to a few tens $M_{\odot} \mathrm{yr}^{-1}$; for each galaxy, we have UV, optical, and, in some cases, near-IR SEDs obtained from large-aperture spectroscopy (Calzetti, Kinney, \& Storchi-Bergmann 1994; McQuade, Calzetti, \& Kinney 1995; Storchi-Bergmann, Kinney, \& Challis 1995; Calzetti, Kinney, \& Storchi-Bergmann 1996); the dust reddening properties of these galaxies in the wavelength range 0.12-2.2 $\mu \mathrm{m}$ are well characterized (Calzetti, Kinney, \& Storchi-Bergmann 1994; Calzetti 1997a). From this original sample, we selected a subsample of 16 objects with positive $I R A S$ detections at $60 \mu \mathrm{m}$ and with more than $50 \%$ of both the blue light and the $\mathrm{H} \alpha$ emission included within the aperture of the UV-optical observations. The last requirement minimizes aperture mismatches between the short- (UV, optical, and near-IR) and long- (FIR) wavelength observations. Visibility constraints and observing time limitations reduced the final number of targets to the eight listed in Table 1. 
TABLE 1

Properties of the Galaxy SAMPle

\begin{tabular}{|c|c|c|c|c|c|c|}
\hline Galaxy Name & $\begin{array}{c}D^{\mathrm{a}} \\
(\mathrm{Mpc})\end{array}$ & $\begin{array}{c}\langle d\rangle^{\mathbf{b}} \\
(\operatorname{arcmin})\end{array}$ & $L_{\mathrm{ap}}(\mathrm{H} \alpha) / L_{\mathrm{tot}}(\mathrm{H} \alpha)^{\mathrm{c}}$ & $E(B-V)^{\mathrm{d}}$ & $\begin{array}{c}\mathrm{SFR}^{\mathrm{e}} \\
\left(M_{\odot} \mathrm{yr}^{-1}\right)\end{array}$ & $\beta^{\mathrm{f}}$ \\
\hline NGC $6090 \ldots \ldots \ldots \ldots$ & 175 & 0.58 & 0.96 & $0.60 \pm 0.06$ & 55.3 & -0.78 \\
\hline NGC $7673 \ldots \ldots \ldots \ldots$ & 68 & 1.25 & 0.85 & $0.41 \pm 0.11$ & 4.3 & -1.48 \\
\hline NGC $5860 \ldots \ldots \ldots \ldots$ & 108 & 0.54 & 0.82 & $0.65 \pm 0.15$ & 8.1 & -0.98 \\
\hline IC $1586 \ldots \ldots \ldots \ldots \ldots$ & 116 & 0.31 & 0.90 & $0.50 \pm 0.09$ & 6.4 & -0.71 \\
\hline Tol $1924-416 \ldots \ldots \ldots$ & 57 & 0.63 & 0.93 & $0.02 \pm 0.02$ & 3.2 & -2.08 \\
\hline Mrk $66 \ldots \ldots \ldots \ldots \ldots$ & 130 & 0.35 & 0.91 & $0.00 \pm 0.05$ & 1.4 & -1.60 \\
\hline ESO $185-\mathrm{IG} 013 \ldots \ldots$ & 112 & 0.35 & 1.00 & $0.16 \pm 0.10$ & 3.7 & -1.38 \\
\hline UGCA $410 \ldots \ldots \ldots \ldots$ & 15 & 0.25 & 0.83 & $0.12 \pm 0.10$ & 0.1 & -1.15 \\
\hline
\end{tabular}

${ }^{\mathrm{a}} \mathrm{H}_{0}=50 \mathrm{~km} \mathrm{~s}^{-1} \mathrm{Mpc}^{-1}$.

b Average angular size.

c Fraction of the total $\mathrm{H} \alpha$ light contained in the UV-optical-near-IR observational aperture. Except for NGC 7673 , the $\mathrm{H} \alpha$ fraction is similar to that of $B$-band light; for NGC 7673, the $B$-band fraction is 0.48 .

d The color excess $E(B-V)$, derived from the ratio of nebular line emissions (Calzetti et al. 1994, 1996). The values are either the average $E(B-V)$ from both $\mathrm{H} \alpha / \mathrm{H} \beta$ and $\mathrm{H} \beta / \mathrm{Br} \gamma$ or the $E(B-V)$ from the ratio $\mathrm{H} \alpha / \mathrm{H} \beta$ alone. In all cases, corrections for the stellar absorption underlying the line emission have been included (Calzetti et al. 1994). Where two nebular line ratios have been used, the dispersion on the average $E(B-V)$ is smaller or at most comparable to the random error.

e The star formation rate, derived from the reddening-corrected $\mathrm{H} \alpha$ flux, assuming a Salpeter initial mass function in the mass range $0.1-100 M_{\odot}$

${ }^{\mathrm{f}}$ Power-law slope of the stellar continuum emission, $F(\lambda) \propto \lambda^{\beta}$, in the range $0.12-0.26 \mu \mathrm{m}$.

The eight objects have redshifts $z<0.03$, metallicities in the range $0.1-2 Z_{\odot}$, intrinsic color excess in the range $E(B-V)=0-0.7 \mathrm{mag}$, and star formation rates (SFRs) in the range $0.2-55 M_{\odot} \mathrm{yr}^{-1}$, as derived from the $\mathrm{H} \alpha$ emission for a $0.1-100 M_{\odot}$ Salpeter initial mass function (IMF). The galaxies are noninteracting, although some display double luminous peaks in their nuclei, perhaps a sign that they are caught at some stage of a merging process. For all eight objects, between $82 \%$ and $96 \%$ of the $\mathrm{H} \alpha$ emission is contained within the UV-optical observational aperture, and for seven of them more than $80 \%$ of the $B$-band emission is included in the aperture; for NGC 7673, the $B$-band fraction in the aperture is $50 \%$. These values have been derived from the literature or, for the $\mathrm{H} \alpha$ emission of NGC 7673, from new imaging data (C. Conselice 1999, private communication). For each galaxy, Table 1 reports the general properties: distance, average angular size, intrinsic optical color excess, SFR, and UV spectral slope $\beta$ [defined as $F(\lambda) \propto \lambda^{\beta}$; see Calzetti et al. 1994]. Table 2 lists the photometry at $12,25,60$, and $100 \mu \mathrm{m}$ from $I R A S$, as well as the photometry at 150 and $205 \mu \mathrm{m}$ from our ISO observations.

The ISO data were obtained with the ISOPHOT instrument and the C200 camera with the 135 and the 200 filters, centered at $\sim 150$ and $\sim 205 \mu \mathrm{m}$, respectively (ISOPHOT Observer's Manual 1994). Sparse maps were obtained in each filter, with one on-source and two off-source exposures per target. The strategy of the double off-source observation was adopted to average out the uneven FIR background, as inferred from the IRAS maps. The background positions were located $\sim 8^{\prime}-10^{\prime}$ away from the target (equivalent to about 3 times the $\mathrm{C} 200$ field of view), on blank-sky positions. The two off-source exposures were always taken before the on-source exposure, to avoid transients. Typical exposure times ranged between 32 and $128 \mathrm{~s}$.

The data reduction and calibration were performed with the PIA ${ }^{2}$ package at the Infrared Processing and Analysis Center (IPAC), following the standard procedure for point sources. This is appropriate for our galaxies which are much smaller than the field of view of the C200 camera $\left(\sim 3^{\prime}\right.$; see Table 1). Data reduction and calibration included correction for the nonlinear response of the detectors, readout deglitching, linear fitting of the signal ramps, resetting of all ramp slopes to a common time interval, flagging of the

${ }^{2}$ PIA is a joint development by the ESA Astrophysics Division and the ISOPHOT Consortium.

TABLE 2

FAR-INFRARED MEASUREMENTS

\begin{tabular}{|c|c|c|c|c|c|c|c|c|}
\hline Galaxy Name & $\begin{array}{l}F(12)^{\mathrm{a}} \\
(\mathrm{Jy})\end{array}$ & $\begin{array}{c}F(25)^{\mathrm{a}} \\
(\mathrm{Jy})\end{array}$ & $\begin{array}{l}F(60)^{\mathrm{a}} \\
(\mathrm{Jy})\end{array}$ & $\begin{array}{c}F(100)^{\mathrm{a}} \\
(\mathrm{Jy})\end{array}$ & $\begin{array}{c}F(150)^{\mathrm{a}} \\
(\mathrm{Jy})\end{array}$ & $\begin{array}{c}F(205)^{\mathrm{a}} \\
(\mathrm{Jy})\end{array}$ & $\begin{array}{c}F(40-120)^{\mathrm{b}} \\
\left(\mathrm{ergs} \mathrm{s}^{-1} \mathrm{~cm}^{-2}\right)\end{array}$ & $\begin{array}{c}F(40-240)^{\mathrm{b}} \\
\left(\mathrm{ergs} \mathrm{s}^{-1} \mathrm{~cm}^{-2}\right)\end{array}$ \\
\hline NGC 6090 & $0.26 \pm 0.02$ & $1.11 \pm 0.04$ & $6.66 \pm 0.26$ & $8.94 \pm 0.40$ & $8.67 \pm 2.15$ & $4.48 \pm 1.12$ & $(32.9 \pm 1.9) \mathrm{E}-11$ & $(43.8 \pm 4.6) \mathrm{E}-11$ \\
\hline NGC 7673 & $0.13 \pm 0.03$ & $0.52 \pm 0.05$ & $4.91 \pm 0.34$ & $6.89 \pm 0.50$ & $7.60 \pm 1.90$ & $4.09 \pm 1.02$ & $(24.6 \pm 2.5) \mathrm{E}-11$ & $(34.2 \pm 4.5) \mathrm{E}-11$ \\
\hline NGC 5860 & $0.13 \pm 0.02$ & $0.20 \pm 0.02$ & $1.64 \pm 0.08$ & $3.02 \pm 0.20$ & $2.63 \pm 0.65$ & $0.72 \pm 0.30$ & $(9.1 \pm 0.7) \mathrm{E}-11$ & $(12.1 \pm 1.4) \mathrm{E}-11$ \\
\hline IC $1586 \ldots$. & $<0.12$ & $<0.21$ & $0.96 \pm 0.08$ & $1.69 \pm 0.20$ & $2.11 \pm 0.52$ & $0.81 \pm 0.37$ & $(5.3 \pm 0.7) \mathrm{E}-11$ & $(7.7 \pm 1.2) \mathrm{E}-11$ \\
\hline Tol $1924-416 \ldots \ldots \ldots$ & $<0.07$ & $0.42 \pm 0.05$ & $1.69 \pm 0.10$ & $1.01 \pm 0.20$ & $0.68 \pm 0.17$ & $0.09 \pm 0.07$ & $(6.8 \pm 0.8) \mathrm{E}-11$ & $(7.7 \pm 1.0) \mathrm{E}-11$ \\
\hline Mrk 66 & $<0.10$ & $0.11 \pm 0.02$ & $0.54 \pm 0.04$ & $0.80 \pm 0.10$ & $0.77 \pm 0.19$ & $<0.18$ & $(2.8 \pm 0.4) \mathrm{E}-11$ & $<3.6 \mathrm{E}-11$ \\
\hline ESO $185-I G 013 \ldots \ldots$ & $<0.07$ & $<0.17$ & $0.42 \pm 0.09$ & $<2.06$ & $0.45 \pm 0.16$ & $<0.30$ & $<4.0 \mathrm{E}-11$ & $<4.5 \mathrm{E}-11$ \\
\hline UGCA $410 \ldots \ldots \ldots \ldots$ & $<0.06$ & $0.10 \pm 0.02$ & $0.30 \pm 0.03$ & $<0.64$ & $0.12 \pm 0.18$ & $<0.21$ & $<1.8 \mathrm{E}-11$ & $<2.0 \mathrm{E}-11$ \\
\hline
\end{tabular}

${ }^{\text {a }}$ Flux densities at 12, 25, 60, and $100 \mu \mathrm{m}$ from IRAS and at 150 and $205 \mu \mathrm{m}$ from our observations with the $I S O$ photometer. The $1 \sigma$ uncertainties are also reported.

$\mathrm{b}$ Total fluxes in the 40-120 $\mu \mathrm{m}$ window from the IRAS data and in the 40-240 $\mu \mathrm{m}$ window from the combination of IRAS and ISO data, with $1 \sigma$ uncertainties. 

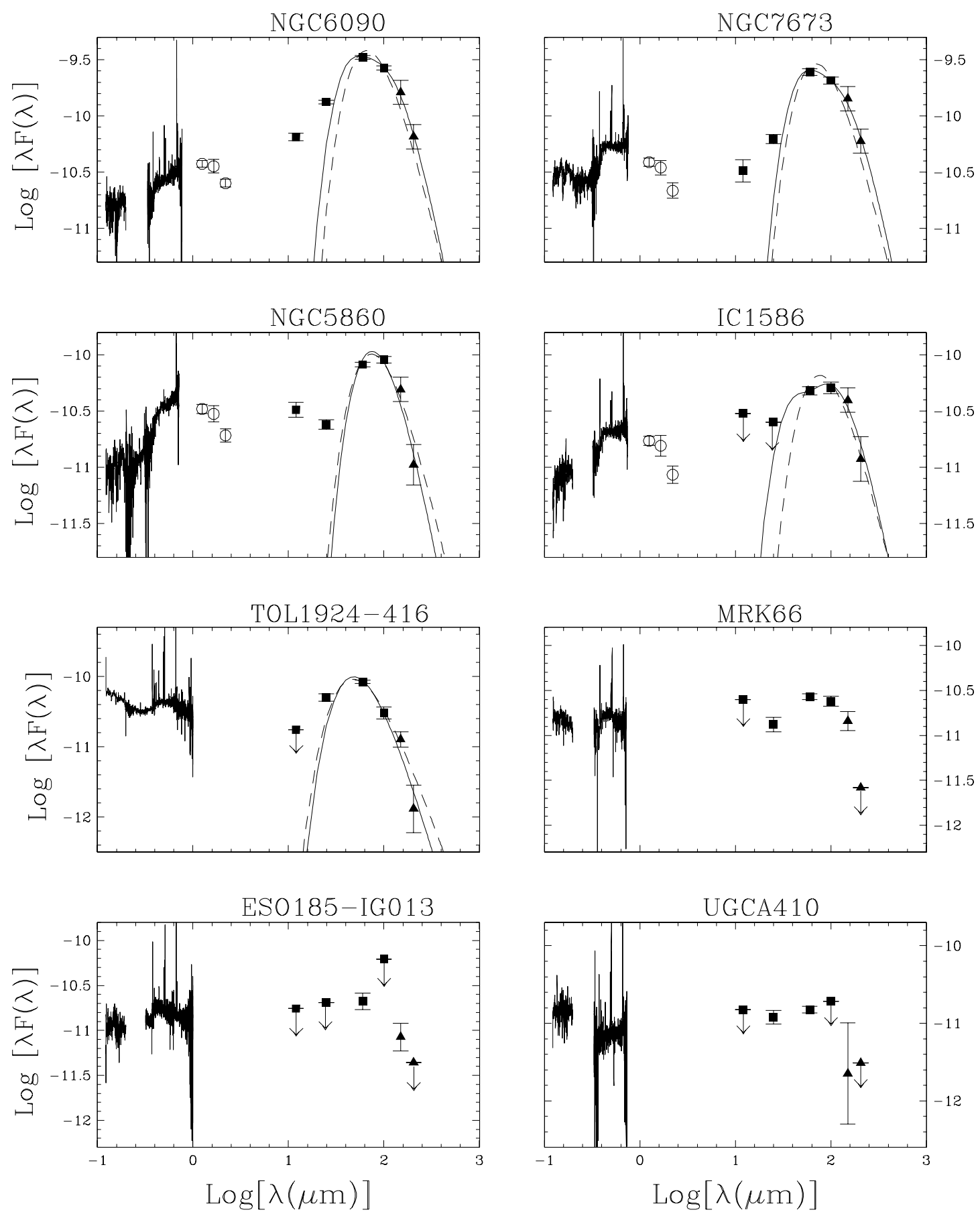

Fig. 1. - Spectral energy distributions, from 0.12 to $240 \mu \mathrm{m}$, of the eight galaxies in our sample. UV-optical spectrophotometry is shown as a continuous line in the wavelength range $0.12-1.0 \mu \mathrm{m}$, near-IR photometry as empty circles, IRAS data as filled squares, and our ISO measurements as filled triangles. Upper limits are shown as downward arrows. No renormalization between the small UV-optical-near-IR observational apertures $\left(\sim 20^{\prime \prime} \times 10^{\prime \prime}\right)$ and the large FIR apertures ( a few arcminutes) has been performed. For five of the galaxies, the best-fit curve to the dust emission in the range $40-240 \mu \mathrm{m}$ is also shown (solid line). The best-fit model corresponds to single or two modified Planck functions with the temperatures given in Table 3 , a dust emissivity $\epsilon=2$, and $50 \%$ contribution to the observed $F(25)$ flux density. For comparison, a model of a single blackbody with $\epsilon=1$ dust emissivity is also shown (dashed line).

deviant slopes, dark current subtraction, average of all the ramp slopes for each pixel, and power calibration against the reference lamp. After extraction of the flux densities, the two off-source exposures were averaged and subtracted from the on-source exposure. Finally, the source signal in the 4 pixels of the $\mathrm{C} 200$ was added together and divided by the fraction of the point-spread function (PSF) included within the $\mathrm{C} 200$ field of view. ISOPHOT absolute calibrations have systematic uncertainties of about $25 \%-30 \%$, which are the dominant source of uncertainty in our data for well-detected galaxies. The $205 \mu \mathrm{m}$ measurements of NGC 5860 and IC 1586 are exceptions: in each case the two backgrounds yielded very different values, and this difference dominates the uncertainty attributed to the detection. For faint detections or upper limits, statistical uncertainties dominate. Table 2 reports the largest among the various sources of uncertainty for the $F(150)$ and $F(205)$ flux densities. Color corrections due to dust temperature variations 
are discussed in $\S 3.1$ and are not included in the flux density values of Table 2 .

Long-wavelength ISO measurements for one of our galaxies, NGC 6090, have already appeared in the literature (Acosta-Pulido et al. 1996). A recent recalibration of the published data (J. Acosta-Pulido 1998, private communication) gives $F(150)=8.26 \mathrm{Jy}$ and $F(205)=7.16 \mathrm{Jy}$. While Acosta-Pulido et al.'s and our determinations of the $F(150)$ flux agree well within $1 \sigma$, the two $F(205)$ flux density determinations differ by $3 \sigma$. For the data of AcostaPulido et al., there is no independent measurement of the background, which may be the source of the discrepancy, because the long-wavelength PSFs fill the C200 field of view. For reference, the average background values we measure around NGC 6090 are $8.9 \pm 0.4 \mathrm{MJy} \mathrm{sr}^{-1}$ at 150 $\mu \mathrm{m}$ and $4.1 \pm 0.4 \mathrm{MJy} \mathrm{sr}^{-1}$ at $205 \mu \mathrm{m}$, where the quoted uncertainties are statistical only.

The last two columns of Table 2 list for each galaxy the integrated FIR fluxes in the ranges $40-120$ and $40-240 \mu \mathrm{m}$, respectively. The flux in the range $40-120 \mu \mathrm{m}$ is from the IRAS 60 and $100 \mu \mathrm{m}$ measurements, using the formula of Lonsdale-Persson \& Helou (1987); the flux in the range $120-240 \mu \mathrm{m}$ is derived from our ISO photometry, after approximating the filter bandpasses to square-equivalent filters of $60 \mu \mathrm{m}$ width centered at 150 and $\sim 205 \mu \mathrm{m}$, respectively (ISOPHOT Observer's Manual 1994), and after including the color corrections discussed in $\S 3.1$. The observed SEDs of the eight galaxies are shown in Figure 1. The following sections concentrate on the five galaxies with positive ISOPHOT detections at both 150 and $205 \mu \mathrm{m}$ and contain no further discussion on the three galaxies undetected at $205 \mu \mathrm{m}$.

\section{ANALYSIS AND RESULTS}

\subsection{Modeling the Emission from Large Dust Grains}

The combination of IRAS and ISO photometry directly measures the FIR emission in the wavelength region 8-240 $\mu \mathrm{m}$. In this section, we model the long-wavelength $(\lambda>40$ $\mu \mathrm{m})$ FIR emission with the goal of obtaining a reliable extrapolation of the dust emission beyond $240 \mu \mathrm{m}$ and an estimate of the contribution from cool dust $(T<25-30 \mathrm{~K})$ to the total FIR luminosity. For this purpose, we assume that the emission longward of $40 \mu \mathrm{m}$ is fully accounted for by large grains in thermal equilibrium with their environment. Modeling of the emission below $\sim 40 \mu \mathrm{m}$ is not attempted because of its complex characteristics (see $\S 1$ ).

A range of parameters is explored for fitting the four data points with $\lambda>40 \mu \mathrm{m}$ : the emission is modeled with a single and with a combination of two modified Planck functions, and the dust emissivity index is allowed to have values $\epsilon=1$ and $\epsilon=2$. This approach is not new (e.g., Chini et al. 1986a; Lonsdale-Persson \& Helou 1987; see also Rowan-Robinson \& Crawford 1989 and some of the references listed in $\S 1$ ) and does not account in detail for the continuum of temperatures and physical conditions of the dust in galaxies but is nevertheless adequate for describing their long-wavelength FIR emission. Through $\chi^{2}$ minimization, the best fit for the dust temperature(s) and, in the case of two modified Planck function, for the fraction of thermal FIR emission contributed by each dust component is searched. The problem is well constrained, as the number of free parameters is less than or equal to the number of independent data points, that is, there are two and four free parameters in the single and the two modified Planck function models, respectively. One additional constraint to the fit is given by the $F(25)$ flux density: the $F(25) / F(60)$ ratios of our galaxies range between 0.11 and 0.25 , implying that between $\sim 25 \%$ and $\sim 75 \%$ of the $F(25)$ flux is contributed by large grains (Boulanger et al. 1988). In the singleblackbody model, the temperature is allowed to vary between 10 and $80 \mathrm{~K}$. For the two-blackbody model, the warm dust temperature may vary between 30 and $80 \mathrm{~K}$, while the cool dust temperature varies between 10 and 30 $\mathrm{K}$. For the temperature range of interest, color corrections to the flux densities at $60,100,150$, and $205 \mu \mathrm{m}$ are about $1.02,1.03,1.16$, and 1.09, respectively, and have been applied to the observed values. The robustness of our fitting routine has been tested against other authors' results for the dust emission of FIR-bright galaxies (Klaas et al. 1997; Colbert et al. 1999); under the same assumptions for the dust emission model, we reproduce the published best-fit temperatures within $3 \mathrm{~K}$.

For three of the galaxies in our sample (NGC 6090, NGC 7673, and IC 1586), the combination of two modified Planck functions yields the best fit to the observed FIR SEDs, with a reduced $\chi^{2}$ in the range $1.1-2.2$. The warm dust component contributes between $50 \%$ and $75 \%$ of the F(25) flux. For NGC 5860 and Tol 1924-416, a single modified Planck function is the best model to describe the long-wavelength emission from the galaxies. In all five galaxies, a dust emissivity index $\epsilon=2$ is a better fit to the FIR SED than $\epsilon=1$, with a factor of $\sim 1.2-1.5$ improvement in the reduced $\chi^{2}$. The first five panels of Figure 1 show the best-fit curves for the five galaxies and, for comparison, a fit to the data using a single blackbody with dust emissivity index $\epsilon=1$ and temperature in the range $38-61 \mathrm{~K}$ (depending on the galaxy). Table 3 lists the temperatures of the warm and cool components, the fraction of the thermal FIR luminosity contributed by each component, and the associated dust masses (see $\S 3.2$ ). Table 4 lists the thermal FIR flux in the range $1-1000 \mu \mathrm{m}$ contributed by the warm + cool dust $\left[F_{t h}(1-1000)\right]$ and the total FIR flux contributed by both large and small grains at any temperature $[F(1-1000)] . F_{\text {th }}(1-1000)$ is calculated by adding the best-fit model fluxes in the ranges $1-40$ and $240-1000 \mu \mathrm{m}$ to the observed flux in the range $40-240 \mu \mathrm{m}$ (Table 2). In $F(1-$ $1000)$, the best-fit model fluxes in the range $8-40 \mu \mathrm{m}$ are replaced with the observational data. Below $8 \mu \mathrm{m}$, we estimate that the contribution of the hot dust and small grain emission to $F(1-1000)$ is less than $\approx 5 \%-10 \%$ of the total and is neglected. Table 4 also gives the ratio of the $F(240$ 1000) flux to the thermal FIR emission. In all five cases considered, this contribution is no more than a few percent, suggesting that the IRAS+ISO measurements detect the bulk of the FIR emission from starburst galaxies. This neglects the potential presence of dust with temperatures $T \lesssim 15$, but the flux contribution of these additional components to the FIR emission is not expected to be large.

In NGC 6090, NGC 7673, and IC 1586, the contribution to the FIR flux of dust at $T \sim 20-23 \mathrm{~K}$ is between $\frac{1}{3}$ and $\frac{2}{3}$ of the FIR emission, a nonnegligible fraction of the total; the rest is contributed by warm dust at temperatures in the range $T \sim 40-55 \mathrm{~K}$. For the two other galaxies, NGC 5860 and Tol 1924-416, the best-fit models give temperatures $T \sim 32$ and $T \sim 50 \mathrm{~K}$, respectively. NGC 5860 does not have a cool component (according to our definition), but its "warm" dust has a fairly low temperature, that is, it is the 
TABLE 3

Dust TeMPeratures AND MASSES

\begin{tabular}{|c|c|c|c|c|c|c|c|c|c|}
\hline Galaxy Name & $\begin{array}{l}T_{w}{ }^{a} \\
(\mathrm{~K})\end{array}$ & $\begin{array}{c}T_{c}{ }^{\mathrm{a}} \\
(\mathrm{K})\end{array}$ & $f_{w}{ }^{\mathrm{b}}$ & $f_{c}{ }^{b}$ & $\begin{array}{l}M_{w}{ }^{c} \\
\left(M_{\odot}\right)\end{array}$ & $\begin{array}{c}M_{c}^{c} \\
\left(M_{\odot}\right)\end{array}$ & $M_{c} / M_{w}{ }^{\mathrm{d}}$ & $M_{w+c} / M_{1 T}^{\mathrm{e}}$ & $\mathrm{H} \mathrm{I} /$ dust $^{\mathrm{f}}$ \\
\hline NGC $6090 \ldots . . . . .$. & $49_{-7}^{+5}$ & $23_{-2}^{+1}$ & $0.59_{+0.14}^{-0.07}$ & $0.41_{-0.14}^{+0.07}$ & $2.33 \mathrm{E}+6$ & $1.03 \mathrm{E}+8$ & 44 & 2.5 & 140 \\
\hline NGC $7673 \ldots \ldots \ldots$ & $43_{-4}^{+6}$ & $20 \pm 2$ & $0.67 \pm 0.11$ & $0.33 \pm 0.11$ & $5.95 \mathrm{E}+5$ & $2.26 \mathrm{E}+7$ & 38 & 3.9 & 360 \\
\hline NGC $5860 \ldots \ldots \ldots .$. & $32 \pm 0$ & $\ldots$ & $1.00 \pm 0$ & $\overline{0}$ & $3.79 \mathrm{E}+6$ & $\ldots$ & 0 & 0.5 & $\ldots$ \\
\hline IC $1586 \ldots \ldots \ldots \ldots$ & $54_{-8}^{+7}$ & $23_{-0}^{+1}$ & $0.40_{+0.06}^{-\overline{0} .05}$ & $0.60_{-0.06}^{+0.05}$ & $7.74 \mathrm{E}+4$ & $1.15 \mathrm{E}+7$ & 149 & 1.9 & 700 \\
\hline Tol $1924-416 \ldots \ldots$ & $50_{-5}^{+0}$ & $31 \pm 0$ & $0.99_{+0.01}^{-0.00}$ & $0.01_{-0.01}^{+0.00}$ & $6.73 \mathrm{E}+4$ & $8.77 \mathrm{E}+3$ & 0.1 & 0.4 & $\cdots$ \\
\hline
\end{tabular}

${ }^{a}$ Best-fit temperatures of the warm $\left(T_{w}\right)$ and cool $\left(T_{c}\right)$ dust components. The two components are modeled as modified Planck functions with dust emissivity index $\epsilon=2$. The reported values refer to the case that $50 \%$ of the $25 \mu \mathrm{m}$ flux is contributed by the thermal (warm + cool) emission. The superscript (subscript) shows the value to add to (subtract from) the mean temperature if $75 \%(25 \%)$ of the $25 \mu \mathrm{m}$ flux is contributed by the thermal emission.

${ }^{\mathbf{b}}$ The fraction of thermal FIR luminosity contributed by the warm $\left(f_{w}\right)$ and the $\operatorname{cool}\left(f_{c}\right)$ dust components. Mean values, superscripts, and subscripts follow the same convention of footnote a.

${ }^{\mathrm{c}}$ The masses associated with the warm and cool dust components, using the mean values in the preceding columns.

${ }^{\mathrm{d}}$ Ratio of the cool to warm dust mass.

e The ratio between the warm + cool dust mass produced by the two-blackbody fit with dust emissivity index $\epsilon=2$ and the dust mass produced by a single-blackbody fit with emissivity index $\epsilon=1$.

${ }_{\mathrm{f}}^{\mathrm{f}}$ The H I-to-dust ratio. The H I mass measurements are from the RC3 (de Vaucouleurs et al. 1991) corrected for 10\% He abundance. The dust mass is the sum of the cool and warm components.

coolest among the galaxies in our sample. Conversely, Tol $1924-416$ is the only object with a high-temperature warm dust component without a substantial cool dust component. Even taking into account the $1 \sigma$ uncertainties of the ISO data, very little, if any, FIR contribution from dust cooler than $\sim 50 \mathrm{~K}$ can be accommodated for this galaxy, unless the dust is colder than $\sim 15 \mathrm{~K}$ (which is beyond the sensitivity range of our ISO observations).

\subsection{Dust Masses and Gas-to-Dust Ratios}

Dust masses can be estimated from long-wavelength FIR fluxes using fairly standard recipes (Hildebrand 1983; Lonsdale-Persson \& Helou 1987). We adopt the formulation of Young et al. (1989):

$$
M_{\text {dust }}=C S_{100} D^{2}\left[\exp \left(143.88 / T_{\text {dust }}\right)-1\right],
$$

where $M_{\text {dust }}$ is in solar masses, $S_{100}$ is the observed flux at $100 \mu \mathrm{m}$ in Jy, $D$ is the galaxy distance in megaparsecs, and the expression in square brackets is the temperaturedependent part of the blackbody emission. The constant $C$ is a combination of fundamental constants and of parameters which depend on the physics of the dust grains, with value $\sim 1.2 M_{\odot} \mathrm{Jy}^{-1} \mathrm{Mpc}^{-2}$ for $\epsilon=2$ and $\sim 6 M_{\odot} \mathrm{Jy}^{-1}$ $\mathrm{Mpc}^{-2}$ for $\epsilon=1$ (Hildebrand 1983; Draine \& Lee 1984).
Table 3 lists the dust masses associated with the warm and cool dust components from the previous section.

In NGC 6090, NGC 7673, and IC 1586, the cool dust mass is between 40 and 150 times the warm dust mass, and the total mass in dust is between $10^{7}$ and $10^{8} M_{\odot}$. The corresponding $\mathrm{H}$ I-to-dust ratios, corrected for $10 \% \mathrm{He}$ abundance, are listed in the last column of Table 3. For reference, typical H I-to-dust ratios are 100-150 for the Milky Way (e.g., Sodroski et al. 1997). If the $\mathrm{H}_{2}$ gas mass is included in the balance (Young et al. 1995), the gas-to-dust ratio of NGC 6090 is $\approx 1.7$ times larger than the Milky Way average (Bohlin, Savage, \& Drake 1978). The two galaxies have similar metallicity, thus they are expected to have approximately similar gas-to-dust ratios; this is what is observed, within the uncertainties of the various estimates. NGC 7673 and IC 1586 have about half the metal content of the Milky Way (e.g., Calzetti et al. 1994), implying that their gas-to-dust ratios are expected to be a factor of 2 larger than the Galaxy value for similar dust depletion patterns. Using upper limits on the CO luminosity of NGC 7673 (Gordon, Heidmann, \& Epstein 1982) to estimate its $\mathrm{H}_{2}$ gas content, we find (gas/dust) $)_{\mathrm{NGC}} 7673 \sim 2$ (gas/dust) ${ }_{\mathrm{MW}}$, as expected from the metallicity differences. For IC 1586, there are no estimates on the $\mathrm{H}_{2}$ mass in the literature; we find $(\mathrm{H} \mathrm{I} / \text { dust })_{\mathrm{IC}} 1586 \sim 4-5(\mathrm{H} \mathrm{I} / \text { dust })_{\mathrm{MW}}$, which is about a

TABLE 4

INFRARED LUMINOSITIES AND BOLOMETRIC CORRECTIONS

\begin{tabular}{|c|c|c|c|c|}
\hline Galaxy Name & $\begin{array}{c}F_{\text {th }}(1-1000)^{\mathrm{a}} \\
\left(\operatorname{ergs~s}^{-1} \mathrm{~cm}^{-2}\right)\end{array}$ & $\begin{array}{c}F(1-1000)^{\mathrm{a}} \\
\left(\operatorname{ergs~s}^{-1} \mathrm{~cm}^{-2}\right)\end{array}$ & $F(240-1000) / F_{\text {th }}(1-1000)^{\mathrm{b}}$ & $\mathrm{BC}_{d}^{\mathrm{c}}$ \\
\hline NGC $6090 \ldots . . . .$. & $(53.8 \pm 4.6) \mathrm{E}-11$ & $(58.8 \pm 4.7) \mathrm{E}-11$ & 0.02 & $1.79 \pm 0.18$ \\
\hline NGC $7673 \ldots \ldots \ldots$ & $(39.7 \pm 4.5) \mathrm{E}-11$ & $(42.0 \pm 4.6) \mathrm{E}-11$ & 0.03 & $1.71 \pm 0.26$ \\
\hline NGC $5860 \ldots \ldots \ldots$. & $(12.7 \pm 1.4) \mathrm{E}-11$ & $(16.3 \pm 1.5) \mathrm{E}-11$ & 0.02 & $1.80 \pm 0.22$ \\
\hline IC $1586 \ldots \ldots \ldots \ldots$ & $(9.6 \pm 1.2) \mathrm{E}-11$ & $(9.6 \pm 1.2) \mathrm{E}-11^{\mathrm{d}}$ & 0.03 & $1.81 \pm 0.33$ \\
\hline Tol $1924-416 \ldots \ldots$ & $(10.5 \pm 1.0) \mathrm{E}-11$ & $(11.1 \pm 1.1) \mathrm{E}-11^{\mathrm{d}}$ & $<0.01$ & $1.63 \pm 0.26$ \\
\hline
\end{tabular}

${ }^{\text {a }}$ The FIR flux in the range $1-1000 \mu \mathrm{m}$, for the thermal (warm + cool) dust component $\left[F_{\mathrm{th}}(1-1000)\right]$ and after adding the mid-IR contribution from small-grain emission $[F(1-1000)]$. See $\S 3.1$ for the derivation of $F_{\mathrm{th}}(1-1000)$ and $F(1-1000)$.

${ }^{b}$ The fraction of FIR flux contained in the $240-1000 \mu$ m window relative to the thermal FIR emission.

c The dust emission bolometric correction, $\mathrm{BC}_{d}=F(1-1000) / F(40-120)$, relative to the IRAS 40-120 $\mu$ m emission.

${ }^{d}$ For IC 1586 , the emission in the 8-40 $\mu \mathrm{m}$ wavelength region has been assumed to be thermal only, because the $F(12)$ and $F(25) I R A S$ data of this galaxy are upper limits (Table 2). The same approximation has been applied to the $8-16 \mu \mathrm{m}$ window of Tol $1924-416$, as the reported $F(12)$ flux is an upper limit. 
factor of 2 larger than the expectations, after taking into account metallicity differences with our Galaxy. This is still an acceptable agreement within our uncertainties, although we cannot exclude that a nonnegligible mass of dust colder than $T \sim 15 \mathrm{~K}$ may exist in IC 1586 .

The total dust mass derived from the two-blackbody fit and $\epsilon=2$ emissivity is within a factor of 4 of the dust mass derived from a single-blackbody model with $\epsilon=1$ emissivity (ninth column of Table 3 ). Therefore, either model produces reasonably consistent masses for dust emitting in the temperature range $20-60 \mathrm{~K}$.

\subsection{The Energy Balance}

This section presents a comparison between the measured amount of stellar energy absorbed by dust, represented by the observed FIR emission, and a predicted value of the dust-absorbed stellar energy derived from the UV-tonear-IR SED of each galaxy in our sample. The goal is to establish whether, and within which accuracy, extant methods for UV-to-near-IR dust reddening correction (the "starburst reddening" curve of Calzetti et al. 1994; see also Calzetti 1997a) can also be used to derive the total dust optical depth in UV-bright, starburst galaxies. For this purpose, the predictions on the dust-absorbed stellar light will be derived by applying the starburst reddening curve and the measured color excess values $E(B-V)$ (Table 1) to the observed UV-to-near-IR SEDs of the galaxies.

Four of the five galaxies have data in the wavelength region $0.12-2.2 \mu \mathrm{m}$, and the fifth, Tol 1924-416, has data in the region $0.12-1.0 \mu \mathrm{m}$ from the literature (Kinney et al. 1993; McQuade, Calzetti, \& Kinney 1995; StorchiBergmann, Kinney, \& Challis 1995; Calzetti, Kinney, \& Storchi-Bergmann 1996). As discussed in $\S 2$, the galaxies in the present sample have been chosen to minimize the aperture mismatch between the UV-optical observations (effective area of $\sim 170 \operatorname{arcsec}^{2}$ ) and the FIR photometry (with effective areas of a few square arcminutes). In addition, we have an estimate of the fraction of light falling outside the UV-optical-near-IR observational apertures (fourth column of Table 1), which we use to correct for the residual effect of aperture mismatch. All UV-to-near-IR
SEDs have been extrapolated down to the Lyman break at $0.0912 \mu \mathrm{m}$ using a power-law fit to the UV spectrum. Table 5 lists the observed flux, $F_{o}$, in the waveband $0.09-2.3 \mu \mathrm{m}$ for the five galaxies.

The intrinsic shape of the stellar emission, $F_{i}(\lambda)$, is recovered using the starburst reddening curve $k^{\prime}(\lambda)=A^{\prime}(\lambda) / E_{s}$ $(B-V)$, with the standard formulation (Calzetti et al. 1994; Calzetti 1997b):

$$
F_{i}(\lambda)=F_{o}(\lambda) 10^{0.4 E_{s}(B-V) k^{\prime}(\lambda)}
$$

with $F_{i}(\lambda)$ and $F_{o}(\lambda)$ the intrinsic and observed stellar continuum flux densities, respectively; the color excess of the stellar continuum $E_{s}(B-V)$ is linked to the color excess derived from the nebular gas emission lines $E(B-V)$ (Table 1) via

$$
E_{s}(B-V)=(0.44 \pm 0.03) E(B-V)
$$

(Calzetti 1997b). The expression of $k^{\prime}(\lambda)$ is

$$
\begin{aligned}
& k^{\prime}(\lambda)=2.659(-1.857+1.040 / \lambda)+R_{V}^{\prime}, \\
& 0.63 \mu \mathrm{m} \leq \lambda \leq 2.20 \mu \mathrm{m} ; \\
&= 2.659(-2.156+1.509 / \lambda \\
&\left.-0.198 / \lambda^{2}+0.011 / \lambda^{3}\right)+R_{V}^{\prime}, \\
& 0.12 \mu \mathrm{m} \leq \lambda<0.63 \mu \mathrm{m} .
\end{aligned}
$$

The amount of UV-to-near-IR stellar continuum light absorbed by dust is the difference $F_{i}-F_{o}$ between the intrinsic and the observed emission. If the entire scattering area of the galaxy is contained within the observational aperture, the reddening curve represents mainly an "absorption" curve. Such an assumption is appropriate for our observational configuration, where large apertures are employed, and a large fraction of the light from the galaxy and practically all the light from the starburst are observed. The energy balance between UV-to-near-IR and FIR must include the fraction of ionizing photons $(\lambda<0.0912 \mu \mathrm{m})$

\begin{tabular}{|c|c|c|c|c|c|}
\hline Galaxy Name & $\begin{array}{c}F_{o}(0.09-2.3)^{\mathrm{a}} \\
\left(\operatorname{ergs~s}^{-1} \mathrm{~cm}^{-2}\right)\end{array}$ & $\begin{array}{c}F_{i}(0.09-2.3)^{\mathrm{a}} \\
\left(\mathrm{ergs} \mathrm{s}^{-1} \mathrm{~cm}^{-2}\right)\end{array}$ & $\begin{array}{c}F_{\text {ion }}^{\mathrm{b}} \\
\left(\operatorname{ergs~}^{-1} \mathrm{~cm}^{-2}\right)\end{array}$ & $\Delta F(0-2.3) / F(1-1000)^{\mathrm{c}}$ & $F_{o} / F_{\text {bol }}{ }^{d}$ \\
\hline NGC $6090 \ldots \ldots \ldots .$. & $8.06 \mathrm{E}-11$ & $33.00 \mathrm{E}-11$ & $10.41 \mathrm{E}-11$ & 0.60 & 0.12 \\
\hline NGC $7673 \ldots \ldots \ldots . .$. & $15.81 \mathrm{E}-11$ & $46.67 \mathrm{E}-11$ & $6.43 \mathrm{E}-11$ & 0.98 & 0.29 \\
\hline NGC $5860 \ldots \ldots \ldots . .$. & $7.01 \mathrm{E}-11$ & $31.39 \mathrm{E}-11$ & $6.31 \mathrm{E}-11$ & 2.14 & 0.31 \\
\hline IC $1586 \ldots \ldots \ldots \ldots$ & $4.57 \mathrm{E}-11$ & $15.48 \mathrm{E}-11$ & $3.50 \mathrm{E}-11$ & 1.53 & 0.32 \\
\hline Tol $1924-416 \ldots . .$. & $17.00 \mathrm{E}-11^{\mathrm{e}}$ & $18.10 \mathrm{E}-11$ & $5.44 \mathrm{E}-11$ & 0.46 & 0.58 \\
\hline
\end{tabular}
absorbed by dust, either directly or after they have been reemitted by hydrogen as Ly $\alpha$ photons; this fraction is about $\frac{2}{3}$ of the total, albeit with a large uncertainty (see references in Calzetti et al. 1995). An estimate of the ionizing

TABLE 5

ENERGY BALANCE

a The observed, $F_{o}$, and intrinsic, $F_{i}$, galaxy emission in the range $0.0912-2.3 \mu \mathrm{m} . F_{i}$ is derived from $F_{o}$, using the starburst reddening curve (see text; Calzetti et al. 1994; Calzetti 1997a) and the color excess values of Table 1 to correct for the effects of dust obscuration.

b The intrinsic ionizing flux, derived from the reddening-corrected $\mathrm{H} \alpha$ emission (Leitherer \& Heckman 1995). About 67\% of this flux is absorbed by dust (see text).

c The energy balance, $\Delta F(0-2.3) / F(1-1000)$, between the stellar light absorbed by dust in the UV-to-near-IR and the observed dust FIR emission. $\Delta F(0-2.3)$ is the difference between the intrinsic and observed flux in the wavelength range $0.0-2.3 \mu \mathrm{m}$ and represents the predicted FIR emission. It is assumed that the scattering region is completely included in the observational aperture. The UV-to-near-IR fluxes have been corrected for the fraction of light outside the observational aperture, using the values in the fourth column of Table 1 . The expected value for the predicted-to-observed FIR ratio is unity, i.e., $\Delta F(0-2.3) / F(1-1000)=1$.

d The ratio of the observed UV-to-near-IR emission, $F_{o}$, to the bolometric emission, $F_{\text {bol }}$, defined as the total flux from the UV to the FIR, $F_{o}(0-2.3)+F(1-1000)$. The UV-to-near-IR fluxes have been corrected for aperture losses via the values in the fourth column of Table 1.

${ }^{\mathrm{e}}$ The stellar emission of Tol 1924-416 includes the wavelength range $0.09-1.0 \mu \mathrm{m}$ only. 
energy is obtained from the reddening-corrected $\mathrm{H} \alpha$ flux, using the formula of Leitherer \& Heckman (1995). The predicted dust-absorbed stellar flux is then given by $\Delta F(0-2.3)=F_{i}-F_{o}+0.67 F_{\text {ion }}$. Table 5 lists both the ionizing flux and the "intrinsic" stellar continuum flux $F_{i}$, integrated from 0.09 to $2.3 \mu \mathrm{m}$. The largest uncertainty in the derivation of $\Delta F(0-2.3)$ is in the $R_{V}^{\prime}=A^{\prime}(V) / E_{s}(B-V)$ parameter of the starburst reddening curve. If we were dealing with standard extinction curves (rather than a reddening curve for galaxies), $R_{V}^{\prime}=R_{V}$ would be the total extinction at $V$, which has the value $R_{V}=3.1$ for the Galactic diffuse interstellar medium. For starburst galaxies the exact meaning of $R_{V}^{\prime}$ is less straightforward, since extinction, scattering, and the geometrical distribution of the dust relative to the emitters are all folded together. However, $R_{V}^{\prime}$ can still be described as an effective total obscuration at $V$ (see Calzetti 1997b; Meurer et al. 1999).

Using the value $R_{V}^{\prime}=(4.88 \pm 0.98)$ (Calzetti 1997b) to calculate $\Delta F(0-2.3)$ yields the predicted-to-observed dustabsorbed light ratios $\Delta F(0-2.3) / F(1-1000)$ listed in Table 5. The ratios vary between 0.6 and 2.1, with an average $\langle\Delta F(0-2.3) / F(1-1000)\rangle=1.18$ (logarithmic average) for the four galaxies with nonnegligible reddening at optical wavelengths, i.e., NGC 6090, NGC 7673, NGC 5860, and IC 1586 . In the ideal case that the reddening curve fully recovers the light lost to dust, the ratio $\Delta F(0-2.3) / F(1-1000)=1$ because of energy conservation. This means that, on average, the starburst reddening curve with $R_{V}^{\prime}=4.88$ overpredicts the dust FIR flux by about $18 \%$. In order to decrease the predicted FIR flux by this excess, the effective obscuration at $V$ must be decreased down to

$$
R_{V}^{\prime}=4.05 \pm 0.80 \text {. }
$$

Although this value $R_{V}^{\prime}$ is derived from the average of only four galaxies, we will show in $\S 4.2$ that it has a more general validity, as it can be applied to large samples of star-forming galaxies. It should also be noted that equation (5) is only $1 \sigma$ away from the original value derived by Calzetti (1997b).

The dispersion around the mean for $\langle\Delta F(0-2.3) /$ $F(1-1000)\rangle$ is about a factor of 2 (fifth column of Table 5). Reasons for such dispersion can be varied. In cases where the application of the reddening curve underestimates the FIR flux, like in NGC 6090, some regions of the central starburst may be heavily enshrouded in dust and they give little, if any, contribution to the observed UV-to-near-IR flux. In cases where the reddening curve overpredicts the FIR emission, as for NGC 5860, variations in the geometry of the stellar populations and the dust (e.g., the entire scattering region is not included in the observational aperture) can play a role. A factor of 2 dispersion in the predicted FIR flux of starburst galaxies is, however, remarkably small, given that a single recipe for dust obscuration corrections is being used. Thus, within the limitations of the small sample available to us, the curve recovers the total dust optical depth of UV-bright, starburst galaxies and can be appropriately called an "obscuration curve."

\section{DISCUSSION}

\subsection{Far-Infrared Emission from Local Starburst Galaxies}

Dust emission at wavelengths beyond $120 \mu \mathrm{m}$ (the IRAS limit) represents a nonnegligible fraction of the FIR flux even in "warm" galaxies like starbursts. Table 4 lists the bolometric corrections between the total FIR dust emission in the wavelength window $1-1000 \mu \mathrm{m}$ and the $40-120 \mu \mathrm{m}$
IRAS measurements for the five galaxies in our sample, which has an average

$$
\mathrm{BC}_{d}=\frac{F(1-1000)}{F(40-120)}=1.75 \pm 0.25
$$

The dust bolometric corrections lower to values between 1.4 and 1.8 , with an average of 1.6 , if only the thermal dust emission is included in the balance. Rigopoulou et al. (1996) measured FIR SEDs for a sample of ultraluminous IRAS galaxies using submillimeter observations. Dust bolometric corrections derived from seven galaxies in this sample by Meurer et al. (1999) give $\mathrm{BC}_{d}(40-1000)=1.4 \pm 0.2$ in the wavelength region $40-1000 \mu \mathrm{m}$, coincident with the median value we obtain in the same wavelength range, that is, $\mathrm{BC}_{d}(40-1000)=1.38 \pm 0.22$.

Buat \& Burgarella (1998) derived dust bolometric corrections for actively star-forming galaxies by constraining the dust emission with IRAS data and the millimeter observations of Andreani \& Franceschini (1996). With their calibration, the total-to-IRAS correction for five of the galaxies in Table 1 (NGC 6090, NGC 7673, NGC 5860, IC 1586, and Mrk 66) is around 1.23, and it is $\mathrm{BC}_{d}<1$ for Tol 1924-416. The corrections are on average $\sim 43 \%$ smaller than ours, and the discrepancy increases for increasing deviation of the galaxies' FIR emission from the single-blackbody approximation. This result suggests that millimeter-wavelength observations alone, even when coupled with IRAS data, are inadequate to constrain the long-wavelength FIR emission. Observations in the millimeter regime fall in the RayleighJeans tail for all dust components warmer than a few degrees kelvin and are difficult to use for deriving the actual emission in the submillimeter range (see, e.g., Chini et al. 1986a; Roche \& Chandler 1993).

For the three galaxies in our sample whose thermal FIR SED is modeled by a combination of two modified Planck functions (Table 3), the cool dust component represents between $30 \%$ and $60 \%$ of the thermal FIR emission. This component is very likely heated by a combination of the central starburst and the ISRF. The comparison of NGC 6090 with NGC 7673 provides support for this statement. The SFRs of the two starbursts, as derived from their extinction-corrected $\mathrm{H} \alpha$ luminosities, differ by a factor of $\sim 11$ (Table 1, after including aperture corrections), while their host galaxies differ by less than a factor of 3 in $R$-band brightness, with NGC 6090 being the brighter and more active of the two. If the cool dust were heated mostly by the ISRF, the fraction of FIR luminosity due to warm dust should be larger in NGC 6090 than in NGC 7673 (Young et al. 1989), i.e., $\left(f_{w} / f_{c}\right)_{\mathrm{NGC} 6090} \approx 4\left(f_{w} / f_{c}\right)_{\mathrm{NGC}} 7673$. Instead, $\left(f_{w} / f_{c}\right)_{\mathrm{NGC} 6090} \sim\left(f_{w} / f_{c}\right)_{\mathrm{NGC}} 7673$, implying that most of the cool dust heating in NGC 6090 is due to the starburst itself. A similar conclusion is reached by comparing NGC 6090 with IC 1586 . The dust temperature decreases for increasing distance of the dust grains from the heating source, for increasing dust column density (optical depth) and for increasing mean wavelength of the source SED (Panagia 1978). Because of the exponential dependence of $T$ on the medium's optical depth, large dust opacities reduce the temperature even in the presence of hard radiation from massive stars. Optically thick regions introduce a spread toward low temperatures of the emission spectrum because of dust self-shielding and shielding of dust grains by $\mathrm{H}_{2}$ (e.g., Natta et al. 1981; Mathis, Mezger, \& Panagia 1983 for 
the giant molecular clouds (GMCs) in the Milky Way; Wall et al. 1996 for COBE observations of Orion). Luminous FIR galaxies, like NGC 6090, are candidates for an opaque medium at most wavelengths (Lisenfeld, Isaak, \& Hills 1999). In the environment of luminous and ultraluminous FIR galaxies, the gas densities are large and can reach about 10 times the density of GMCs (Downes \& Solomon 1998); dust column densities are, thus, likely to be large.

Effects of dust optical depth explain qualitatively why Tol 1924-416 does not have a cool dust component and its dust is generally warm, with $T \sim 50 \mathrm{~K}$. Tol $1924-416$ is the most metal-poor and dust-poor galaxy in our sample of five, with a metallicity about 5-10 times lower than the other four and a very small optical dust reddening (fifth column of Table 1). Comparably low dust opacities are expected if the gas column densities are similar among the five galaxies. Dust self-shielding is less effective in Tol 1924-416 than in the other galaxies, and the radiation from the massive stars will heat the dust to relatively high temperatures in the entire starburst region.

Optically thick regions are not the only cause for the presence of a cool dust component; the nonionizing stars produced in the central star-forming region can also heat the dust to relatively low color temperatures, similar in effect to the ISRF. For typical star formation durations of $\sim 50-100 \mathrm{Myr}$ or more, a number of long-living, nonionizing stars are accumulated in the starburst site and become important for the dust heating. Photons from these stars are less energetic on average than the radiation from massive stars and can also travel long distances before being intercepted by a dust grain (Mezger et al. 1982). The dust is then heated to relatively low color temperatures (e.g., the case of the postburst galaxy NGC 4945; Koornneef 1993). For the typical SFRs of NGC 7673, NGC 5860, and IC 1586 (Table 1), the energy density of the starburst's nonionizing stars equals that of the host galaxy diffuse radiation field at a distance about 0.6 times the radius of the galaxy from the starburst center, assuming that the host galaxy has stellar population characteristics similar to the Milky Way. Since $\langle u\rangle \propto T^{4+\epsilon}$, a factor of a few variation in the energy density $\langle u\rangle$ produces only a few percent change in the dust temperature $T$.

Our galaxies are generally classified as "UV bright." However, with the exception of Tol 1924-416, which contains relatively little dust, all the others are affected by dust obscuration in a nonnegligible manner. The energy emerging at UV-to-near-IR wavelengths is $\frac{1}{3}$ or less of the bolometric luminosity; furthermore, the UV emission below 0.2 $\mu \mathrm{m}$ represents less than $15 \%$ of the FIR emission (Tables 5 and 6 and Fig. 1). A "picket fence" or "clumpy" geometry for the dust can account for the apparent contradiction that such galaxies are at the same time "UV bright" and good FIR emitters: most of the UV-to-near-IR emission will emerge from dust-clear lines of sight, while the FIR emission emerges from dusty lines of sight (Calzetti 1997a; Gordon, Calzetti, \& Witt 1997).

\subsection{The Starburst Obscuration Curve: Application to a Larger Sample of Local Galaxies}

The analysis in $\S 3.3$ has shown that the starburst reddening/obscuration curve of Calzetti et al. (1994) measures the total dust opacity affecting the UV-to-near-IR SEDs of starburst galaxies with an accuracy of a factor of about 2 in each individual case. Up to this point, the validity of such a result is limited to the few galaxies in our sample. In this section, the generality of the obscuration curve is tested on a much larger sample of UV-bright, starburst galaxies.

UV data for a sample of 47 actively star-forming galaxies are drawn from the Kinney et al. (1993) IUE Atlas and are complemented with IRAS FIR fluxes. A list of general characteristics of the 47 galaxies is reported by Meurer et al. (1999), together with the values of the UV spectral slopes $\beta$ and a discussion on the uncertainties due to aperture mismatches between IRAS and IUE data. The UV spectral slope $\beta$, derived in the wavelength range $0.12-0.26 \mu \mathrm{m}$, is a measure of the dust reddening affecting the UV stellar continuum of a starburst galaxy (Calzetti et al. 1994).

Because of the highly incomplete spectral information available, basically only UV and IR $A S$ FIR data, the energy balance approach of $\S 3.3$ cannot be applied to the large sample. A more approximate method is therefore employed here (Meurer et al. 1999): in addition to a measure of the UV and optical reddening $[\beta$ and $E(B-V)]$, an estimate of the UV total obscuration is derived for each galaxy, and the two quantities are compared with predictions from the starburst obscuration curve. In a starburst galaxy most of the intrinsic stellar light is emitted in the UV, and the FIR-toUV energy ratio, $F(\mathrm{IR}) / F(\mathrm{UV})$, is an estimator of the global dust optical depth at UV wavelengths (Xu \& Helou 1996). In the present case, $F(\mathrm{IR})=F(40-120)$ and $F(\mathrm{UV})=[0.16$ $f(0.16)]$, with $f(0.16)$ the flux density at $0.16 \mu \mathrm{m}$. The ratio $F(\mathrm{IR}) / F(\mathrm{UV})$ is converted into an obscuration $A(\lambda)$ (in magnitudes) at $0.16 \mu \mathrm{m}$ via

$$
A(0.16) \simeq 2.5 \log \left[\frac{1}{E} \frac{F(\mathrm{IR})}{F(\mathrm{UV})}+1\right],
$$

where

$$
E \simeq 0.9 .
$$

The constant $E$ is the ratio of two quantities: the bolometric correction of the UV-to-near-IR stellar light relative to the

TABLE 6

\begin{tabular}{|c|c|c|c|c|c|c|}
\hline Galaxy Name & $\begin{array}{l}L_{\mathrm{bol}}{ }^{\mathrm{a}} \\
\left(L_{\odot}\right)\end{array}$ & $\lambda f(\lambda)_{0.17} / F_{\text {th }}(\mathrm{FIR})^{\mathrm{b}}$ & $\lambda f(\lambda)_{0.22} / F_{\mathrm{th}}(\mathrm{FIR})^{\mathrm{b}}$ & $\lambda f(\lambda)_{100} / F_{\text {th }}(\mathrm{FIR})^{\mathrm{b}}$ & $\lambda f(\lambda)_{150} / F_{\text {th }}(\mathrm{FIR})^{\mathrm{b}}$ & $\lambda f(\lambda)_{205} / F_{\text {th }}(\mathrm{FIR})^{\mathrm{b}}$ \\
\hline NGC $6090 \ldots \ldots \ldots$. & $6.8 \mathrm{E}+11$ & 0.03 & ... & 0.50 & 0.32 & 0.12 \\
\hline NGC $7673 \ldots \ldots \ldots$ & $9.1 \mathrm{E}+10$ & 0.10 & 0.09 & 0.52 & 0.38 & 0.15 \\
\hline NGC $5860 \ldots \ldots \ldots$ & $9.9 \mathrm{E}+10$ & 0.09 & 0.07 & 0.71 & 0.41 & 0.08 \\
\hline IC $1586 \ldots \ldots \ldots \ldots$ & $6.7 \mathrm{E}+10$ & 0.11 & $\ldots$ & 0.53 & 0.44 & 0.12 \\
\hline Tol $1924-416 \ldots \ldots$ & $3.2 \mathrm{E}+10$ & 0.77 & 0.58 & 0.29 & 0.13 & 0.013 \\
\hline
\end{tabular}

SPECTRAL ENERGY DistribUTIONS

a Bolometric luminosities, in solar units, calculated using the fluxes of Table 5 and the distances and aperture corrections of Table 1.

b The energy coming out at $0.17,0.22,100,150$, and $205 \mu$ m relative to the thermal dust FIR emission. 
UV emission at $0.16 \mu \mathrm{m}$ and the dust bolometric correction relative to the fraction of FIR light detected in the IRAS window. Our value of $E$ is about $30 \%$ smaller than that of Meurer et al. (1999) for the following reasons: (1) the dust bolometric correction used in equation (7) is the one we derive from our ISO observations (eq. [6]), which is about $25 \%$ larger than that used by the other authors; (2) the bolometric correction of the stellar light is derived under the assumption that a star formation event lasts $\approx 10^{8}-10^{9}$ $\mathrm{yr}$ (Calzetti 1997a), rather than $<10^{8} \mathrm{yr}$; (3) the fraction of stellar light absorbed by dust at each wavelength is treated semianalytically, instead of being approximated to its mean value at UV wavelengths. Equation (7) has been derived assuming that the approximation of foreground dust holds for UV-bright starbursts; various observations support this assumption (Calzetti et al. 1994, 1996).

A plot of $A(0.16)$ as a function of the UV spectral slope $\beta$ is shown in Figure 2 for the 47 starburst galaxies. The tight correlation between $A(0.16)$ and $\beta$ shows that the same amount of dust responsible for the UV reddening is also responsible for the total UV opacity (Meurer et al. 1999). The continuous line in Figure 2 is the correlation between $A(0.16)$ and $\beta$ expected from the starburst obscuration curve of $\S 3.3$ :

$$
A(0.16)=2.31\left(\beta-\beta_{o}\right)=2.31 \beta+4.85 .
$$

For the no-dust case $[A(0.16)=0]$, an intrinsic UV spectral slope $\beta_{o}=-2.1$ has been adopted (Calzetti 1997a), as expected for continuous star formation over a timescale of 1 Gyr. Once $\beta_{o}$ is fixed, there are no other free parameters in equation (9). The excellent agreement between the locus of the observed points and the predicted curve suggests that

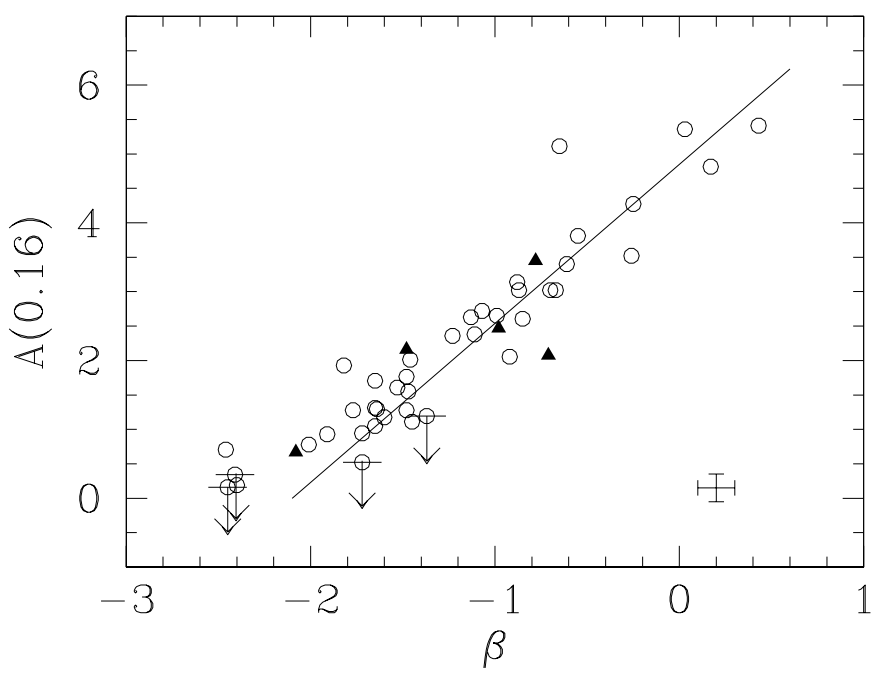

FIG. 2.-Opacity at $0.16 \mu \mathrm{m}, A(0.16)$, plotted as a function of the UV spectral slope $\beta$ for a sample of 47 starburst galaxies from Kinney et al.'s IUE Atlas. The filled triangles mark the position of our five galaxies; upper limits are indicated as downward arrows. A representative $1 \sigma$ error bar is given at the bottom right corner of the diagram. $A(0.16)$ is derived from the ratio FIR $_{I R A S}$ to UV luminosity. $\beta$ is a measure of the dust reddening affecting the galaxy. The tight correlation between the two quantities demonstrates that the same amount of dust responsible for the reddening is also responsible for the total obscuration (see Meurer et al. 1999). The continuous line is the trend predicted by the starburst obscuration curve $k^{\prime}(\lambda)$ (eqs. [4] and [5]). The slope of the line is dictated by the functional shape of $k^{\prime}(\lambda)$, and the intercept by the UV characteristics of the intrinsic stellar SED (see text). the reddening curve of equation (4), with the zero point of equation (5), is a representative obscuration curve for UVbright, star-forming galaxies in general. It recovers fairly accurately (within $\sim 20 \%$ ) the mean dust opacity of a large sample of such galaxies. This is not the result of a circular argument: the reddening curve (eq. [4]) has been derived entirely from UV-to-near-IR data of starburst galaxies, while its zero point (eq. [5]) has been derived from the FIR data of a small sample of five galaxies; the data in Figure 2 use an independent set of data: the FIR emission detected by IRAS for a relatively large number of star-forming galaxies.

For fixed $\beta$, the scatter about the mean value of $A(0.16)$ is typically $0.5-0.6 \mathrm{mag}$ (one sided); thus, a measure of the global opacity of any individual star-forming galaxy using the starburst obscuration curve will give a typical uncertainty of a factor of 1.6-1.7, not very different from the factor of $\sim 2$ found in $\S 3.3$.

For $\beta<-1.4$, the data points of Figure 2 tend to have higher values of $A(0.16)$ than predicted by equation (9). There are two possible reasons for this effect. First, the uncertainty of converting the FIR emission into a UV opacity is large, especially with limited wavelength coverage. Detailed energy balance analysis should be performed on the dust-poor galaxies in order to understand whether the observed underestimate of the opacity is an actual problem or is an artifact of how $A(0.16)$ is constructed. Second, very blue, dust-poor galaxies are generally associated with shorter timescales for star formation (e.g., Calzetti 1997a), implying that, in equation (9), $\beta_{o}$ should be changed from -2.1 to a more negative value to properly represent the data points at $\beta \lesssim-1.4$. For instance, continuous star formation over $0.1 \mathrm{Gyr}$ gives $\beta_{o}=-2.35$ for solar metallicity (Leitherer \& Heckman 1995). Variations in the intrinsic stellar population of each galaxy may thus play a small but not insignificant role in the details of the agreement between data and prediction.

For a subset of $\sim 30$ starburst galaxies, color excess measurements for the ionized gas, $E(B-V)$, are available from Calzetti et al. (1994). Figure 3 shows the obscuration $A(0.16)$ as a function of $E(B-V)$. A correlation between the two quantities is present also in this case, although with a larger scatter than in Figure 2. Formally, equations (4) and (5) imply a correlation between $A(0.16)$ and $E(B-V)$ expressed as

$$
A(0.16)=4.39 E(B-V) .
$$

The predicted correlation marks the lower envelope to the data points of Figure 3. $E(B-V)$ measures the reddening of the ionized gas (Calzetti et al. 1994), while $A(0.16)$ is a measure of the dust obscuration affecting the UV stellar continuum; the two are, therefore, not directly related. The scatter in the relation between $A(0.16)$ and $E(B-V)$ is no worse than the scatter between $\beta$ and $E(B-V)$ found by Calzetti et al. (1994), which is an imprint of the differential reddening between stellar continuum and ionized gas (eq. [3]) and of the variations in the intrinsic stellar population between galaxies. For fixed $E(B-V)$, the scatter about the mean value of $A(0.16)$ is about $1 \mathrm{mag}$ (one sided).

One final caveat: the obscuration curve may not be applicable to very compact, dusty starbursts, where dust optical depths are large (e.g., ultraluminous FIR galaxies) and the foreground dust approximation may not hold. However, the issue is still open. 


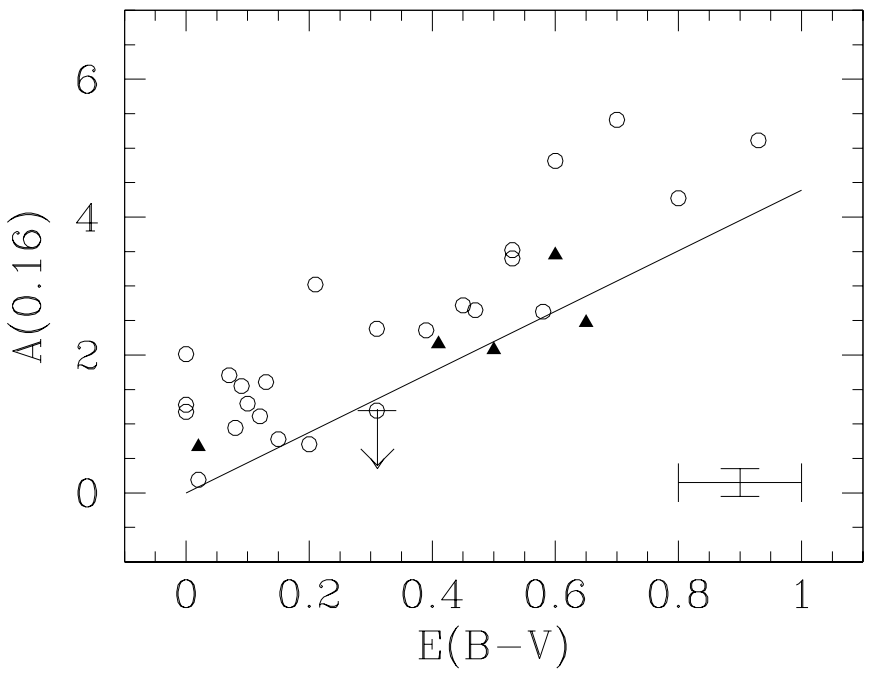

FIG. 3.-Opacity at $0.16 \mu \mathrm{m}, A(0.16)$, plotted as a function of the color excess $E(B-V)$ for a sample of 30 starburst galaxies (Calzetti et al. 1994). $E(B-V)$ is measured from the ionized gas emission. Symbols are as in Fig. 2. A representative $1 \sigma$ error bar is given at the bottom right corner of the diagram. The continuous line shows the trend predicted by the starburst obscuration curve $k^{\prime}(\lambda)$ (eqs. [4] and [5]), after requiring that the opacity of the stellar continuum be zero $[A(0.16)=0]$ if the reddening of the ionized gas is zero $[E(B-V)=0]$. The predicted line marks the lower envelope of the correlation between $A(0.16)$ and $E(B-V)$.

\subsection{Implications for High-Redshift Galaxies}

As mentioned in $\S 1$, the central regions of local UVbright, starburst galaxies show some observational characteristics similar to those of the Lyman-break galaxies at $z>2.5$ (Steidel et al. 1999). In both cases, the objects are active star-forming systems with median star formation rates per unit area of $\approx 1-2 M_{\odot} \mathrm{yr}^{-1} \mathrm{kpc}^{-2}$. Another characteristic they share is the large spread in rest-frame UV colors (Dickinson 1998). As seen in the previous section, the range of UV spectral indices of local starbursts is closely linked to the amount of dust reddening present in the galaxy. Dust reddening has been proposed also as an explanation for the observed range of UV colors of the Lymanbreak galaxies (Calzetti 1997b; Meurer et al. 1997, 1999; Pettini et al. 1998; Steidel et al. 1999).

The median of the distribution of observed UV colors corresponds to a color excess $E_{s}(B-V)=0.15$ for the stellar continuum [Steidel et al. 1999, using the normalization $R^{\prime}(V)=4.88$ of Calzetti 1997b]. The new normalization derived here, $R^{\prime}(V)=4.05$, implies a negligible change to the color excess, $E_{s}(B-V)=0.16$, mainly because the new zero point affects $k^{\prime}(\lambda)$ by less than $8 \%$ at $\lambda \lesssim 0.17 \mu \mathrm{m}$. Thus, the intrinsic UV flux at $0.15-0.17 \mu \mathrm{m}$ is on average 4.1-4.6 times larger than what observed. Dust absorbs about $75 \%-80 \%$ of the UV light in Lyman-break galaxies at $z \approx 3$, a figure compatible with the maximum value of $82 \%-85 \%$ inferred from the cosmological evolution of the dust content of galaxies (Calzetti \& Heckman 1999; Pei, Fall, \& Hauser 1999).

The extended wavelength coverage of our ISO sample can be used to predict the characteristics of the FIR emission from high-redshift, UV-bright, star-forming galaxies. Table 6 lists for our sample galaxies the bolometric luminosity and the energy emitted at a few selected wavelengths normalized to the thermal FIR emission. As already remarked, the bulk of the energy, more than $\frac{2}{3}$ (Table 5), emerges at FIR wavelengths, with the exception of Tol 1924-416, which is the dust-poor galaxy in this sample. Table 7 lists selected observational properties of the same galaxies placed at redshifts $z=1$ and $z=3$, respectively, in a universe with $\Omega_{M}=1, \Omega_{\Lambda}=0$, and $\mathrm{H}_{o}=50 \mathrm{~km} \mathrm{~s}^{-1}$ $\mathrm{Mpc}^{-1}$. At $z=3$, the five galaxies would have observed magnitudes $R \sim 25.5-27$ (rest frame $\lambda \sim 0.17 \mu \mathrm{m}$ ), about 1.0-2.5 mag lower than the $\sim R^{*}$ value determined by Steidel et al. (1999) for the Lyman-break galaxies; the expected flux densities at rest frame 100, 150, and $205 \mu \mathrm{m}$, which correspond to the observer's frame 400,600 , and 820 $\mu \mathrm{m}$, are generally below the detection limits reached by the deepest submillimeter surveys $(\sim 2 \mathrm{mJy}$ for a $5 \sigma$ detection with SCUBA at the James Clerk Maxwell Telescope (JCMT), Hughes et al. 1998). A similar conclusion applies to the $z=1$ case: our galaxies placed at that redshift would have fairly faint $B$ magnitudes (rest frame $0.22 \mu \mathrm{m}$ ) and equally faint FIR flux densities. Tables 8 and 9 give the predicted FIR flux densities and corresponding bolometric luminosities for selected SEDs (NGC 6090, NGC 7673, and Tol 1924-416) covering a range of $B$ or $R$ magnitudes at redshifts $z=1$ and $z=3$, respectively. The predicted FIR fluxes are, where possible, directly from IRAS or ISO measurements whose redshifted wavelengths are the closest to recent submillimeter surveys (e.g., SCUBA: 450 and 850 $\mu \mathrm{m})$. Otherwise, they are extrapolated from the model SEDs.

Both Tables 8 and 9 show that only very bright galaxies, $L_{\text {bol }} \gtrsim(2-3) \times 10^{12} L_{\odot}$, are detectable with the current sensitivity limits of SCUBA for the range of SEDs considered here (see, e.g., the review by Lilly et al. 1999b). These

TABLE 7

High-Redshift Magnitudes AND FluXes

\begin{tabular}{|c|c|c|c|c|c|c|c|c|}
\hline \multirow[b]{2}{*}{ GALAXY NAME } & \multicolumn{4}{|c|}{$z=1$} & \multicolumn{4}{|c|}{$z=3$} \\
\hline & $\begin{array}{c}B^{\mathrm{a}} \\
(A B \mathrm{mag})\end{array}$ & $\begin{array}{c}f(200)^{\mathrm{b}} \\
(\mathrm{mJy})\end{array}$ & $\begin{array}{c}f(300)^{\mathbf{b}} \\
(\mathrm{mJy})\end{array}$ & $\begin{array}{c}f(410)^{\mathrm{b}} \\
(\mathrm{mJy})\end{array}$ & $\begin{array}{c}R^{\mathrm{a}} \\
(A B \mathrm{mag})\end{array}$ & $\begin{array}{c}f(400)^{\mathbf{b}} \\
(\mathrm{mJy})\end{array}$ & $\begin{array}{c}f(600)^{\mathrm{b}} \\
(\mathrm{mJy})\end{array}$ & $\begin{array}{c}f(820)^{\mathrm{b}} \\
(\mathrm{mJy})\end{array}$ \\
\hline NGC $6090 \ldots \ldots \ldots$ & $\ldots$ & $\ldots$ & $\ldots$ & $\ldots$ & 25.7 & 1.88 & 1.80 & 0.92 \\
\hline NGC $7673 \ldots \ldots \ldots$ & 24.7 & 1.26 & 1.38 & 0.75 & 26.8 & 0.21 & 0.23 & 0.13 \\
\hline NGC $5860 \ldots \ldots \ldots$ & 24.9 & 1.84 & 1.60 & 0.43 & 27.1 & 0.25 & 0.21 & 0.06 \\
\hline IC $1586 \ldots \ldots \ldots \ldots$ & $\ldots$ & $\ldots$ & $\ldots$ & $\ldots$ & 27.0 & 0.16 & 0.20 & 0.08 \\
\hline Tol $1924-416 \ldots \ldots$ & 24.5 & 0.13 & 0.09 & 0.01 & 26.4 & 0.02 & 0.01 & $<0.01$ \\
\hline
\end{tabular}

${ }^{a}$ Expected $B$ or $R$ magnitudes, in observer's frame, after redshifting the galaxies to $z=1$ and $z=3$, respectively. The chosen cosmology has $\Omega_{M}=1, \Omega_{\Lambda}=0$, and $\mathrm{H}_{0}=50 \mathrm{~km} \mathrm{~s}^{-1} \mathrm{Mpc}^{-1}$.

${ }^{b}$ Expected FIR fluxes, at the indicated wavelengths in observer's frame, obtained by redshifting the measured fluxes at 100,150 , and $205 \mu \mathrm{m}$ to $z=1$ and $z=3$. 
TABLE 8

Predicted Far-Infrared Flux Densities at REDSHIFT $z=1$

\begin{tabular}{crrc}
\hline \hline $\begin{array}{c}B^{\mathrm{a}} \\
(A B \mathrm{mag})\end{array}$ & $\begin{array}{r}f(410) \\
(\mathrm{mJy})\end{array}$ & $\begin{array}{r}f(850) \\
(\mathrm{mJy})\end{array}$ & $\begin{array}{c}L_{\text {bol }} \\
\left(L_{\odot}\right)\end{array}$ \\
\hline $20.0 \ldots \ldots$ & 56.60 & 11.50 & $6.9 \mathrm{E}+12$ \\
& 0.76 & 0.11 & $2.0 \mathrm{E}+12$ \\
$21.0 \ldots \ldots$ & 22.60 & 4.48 & $2.8 \mathrm{E}+12$ \\
& 0.30 & 0.04 & $8.0 \mathrm{E}+11$ \\
$22.0 \ldots \ldots$ & 8.98 & 1.82 & $1.1 \mathrm{E}+12$ \\
& 0.12 & 0.02 & $3.2 \mathrm{E}+11$ \\
$23.0 \ldots \ldots$ & 3.57 & 0.72 & $4.3 \mathrm{E}+11$ \\
$24.0 \ldots \ldots$. & 0.05 & $<0.01$ & $1.2 \mathrm{E}+11$ \\
& 1.42 & 0.29 & $1.8 \mathrm{E}+11$ \\
& 0.02 & $<0.01$ & $5.1 \mathrm{E}+10$ \\
\hline
\end{tabular}

${ }^{\text {a }}$ For each magnitude $B$, two values (two rows) of the FIR flux density at 410 and 850 $\mu \mathrm{m}$ and of the bolometric luminosity are reported. The two rows give values for galaxies with the SEDs of NGC 7673 and Tol 1924-416, respectively.

sources are similar to or brighter than the typical local ultraluminous FIR galaxy, like Arp 220. The ability to detect the dust FIR emission from a high-redshift galaxy depends, however, not only on the intrinsic luminosity of the source, but also on the details of the dust SED. There is a factor of 5-10 difference between Tol 1924-416 and the other four galaxies in the amount of energy coming out at rest frame $205 \mu \mathrm{m}$ relative to the total thermal FIR emission (see last column of Table 6). For comparison, the 205 $\mu \mathrm{m}$-to-total FIR energy in Arp 220 is also about 2.5-5 times larger than in Tol 1924-416 (from the data of Klaas et al. 1997). The difference is mainly driven by the absence of a cool dust component in Tol 1924-416. As discussed in $\S 4.1$, there is very little dust emission with temperature $T \lesssim 50 \mathrm{~K}$ in Tol 1924-416, independent of the chosen dust emissivity, although this result needs further confirmation.

TABLE 9

Predicted FIR Flux Densities at REDSHIFT $z=3$

\begin{tabular}{crrc}
\hline \hline $\begin{array}{c}R^{\mathrm{a}} \\
(A B \text { mag })\end{array}$ & $\begin{array}{r}f(400) \\
(\mathrm{mJy})\end{array}$ & $\begin{array}{r}f(820) \\
(\mathrm{mJy})\end{array}$ & $\begin{array}{c}L_{\text {bol }} \\
\left(L_{\odot}\right)\end{array}$ \\
\hline $23.0 \ldots \ldots$ & 22.60 & 11.10 & $8.1 \mathrm{E}+12$ \\
& 7.04 & 4.16 & $3.0 \mathrm{E}+12$ \\
& 0.51 & 0.05 & $7.3 \mathrm{E}+11$ \\
$24.0 \ldots \ldots$ & 8.98 & 4.42 & $3.3 \mathrm{E}+12$ \\
& 2.80 & 1.66 & $1.2 \mathrm{E}+12$ \\
& 0.20 & 0.02 & $2.9 \mathrm{E}+11$ \\
$25.0 \ldots \ldots$ & 3.58 & 1.76 & $1.3 \mathrm{E}+12$ \\
& 1.12 & 0.66 & $4.8 \mathrm{E}+11$ \\
& 0.08 & $<0.01$ & $1.1 \mathrm{E}+11$ \\
$26.0 \ldots \ldots$ & 1.42 & 0.70 & $5.1 \mathrm{E}+11$ \\
& 0.44 & 0.26 & $1.9 \mathrm{E}+11$ \\
& 0.03 & $<0.01$ & $4.6 \mathrm{E}+10$ \\
$27.0 \ldots .$. & 0.57 & 0.28 & $1.4 \mathrm{E}+11$ \\
& 0.18 & 0.11 & $7.5 \mathrm{E}+10$ \\
& 0.01 & $<0.01$ & $1.9 \mathrm{E}+10$ \\
\hline
\end{tabular}

a Similar to Table 8 , for each magnitude $R$, three values (three rows) of the FIR flux density at 400 and $820 \mu \mathrm{m}$ and of the bolometric luminosity are reported. The three rows give values for galaxies with the SEDs of NGC 6090, NGC 7673 , and Tol 1924-416, respectively.
We can speculate whether the long-wavelength FIR SEDs of high-redshift galaxies may be expected to be consistent with "purely" warm dust emission and be similar to the Tol 1924-416. Low dust temperatures are the result of high dust column densities and low SFRs, both contributing to lower the number of UV photons available to each dust grain. The dust optical depth of Lyman-break galaxies is not known, but educated guesses can be made with the available information. High-redshift galaxies are expected to be more metal poor than local galaxies $\left(\approx 1 / 10 Z_{\odot}\right.$ at $z \sim$ 3, e.g., Pei \& Fall 1995; Calzetti \& Heckman 1999), although preliminary measurements seem to suggest relatively high abundances in Lyman-break galaxies (De Mello, Leitherer, \& Heckman 1999; Pettini et al. 1999). Low metal abundances do not necessarily imply low dust column densities: high-redshift galaxies may still have a large reservoir of gas, if they have not previously undergone a major star formation phase. Damped Ly $\alpha$ absorption systems at $z \sim 3$ have $\mathrm{H}$ I column densities in the range $N(\mathrm{H} \mathrm{I})=(2-$ 10) $\times 10^{21} \mathrm{~cm}^{-2}$ (Storrie-Lombardi, McMahon, \& Irvin 1996), consistent with the total gas column densities of $\approx 10^{22} \mathrm{~cm}^{-2}$ predicted by models (Calzetti \& Heckman 1999). The result is that the dust optical depth of highredshift galaxies may be as large as that of local starbursts like NGC 7673. However, Lyman-break galaxies have reddening-corrected SFRs about 10 times larger than NGC 7673 itself (Steidel et al. 1999), implying that about 10 times more UV photons are available for each dust grain and that the dust temperature may be as much as $50 \%$ higher than in local starbursts. The possible youth of the stellar populations in the Lyman-break galaxies (ages $\lesssim 1-2$ Gyr) may also argue in favor of warm dust. In conclusion, the dust in high-redshift galaxies may be warmer than in NGC 6090like and NGC 7673-like starbursts and potentially as warm as in Tol 1924-416, implying that the distant galaxies would be undetectable with current submillimeter instrumentation.

\section{SUMMARY AND CONCLUSIONS}

The long-wavelength coverage afforded by ISO has been crucial for characterizing the thermal FIR emission of actively star-forming galaxies. We observed with ISOPHOT eight starburst galaxies at both 150 and $205 \mu \mathrm{m}$ and detected five in both bands. In two of the detected objects, NGC 5860 and Tol 1924-416, the best fit to the IRAS +ISO dust emission in the wavelength range $\sim 40-240 \mu \mathrm{m}$ is given by a single temperature value with dust emissivity index $\epsilon=2$. The dust is warm in both galaxies, $T \sim 32 \mathrm{~K}$ in NGC 5860 and $T \sim 50 \mathrm{~K}$ in Tol 1924-416, but the presence of cold dust with $T \lesssim 15 \mathrm{~K}$ cannot be excluded in either case. In the other three objects (NGC 6090, NGC 7673, and IC 1586), the best fit to the thermal dust emission is given by two modified Planck functions with temperature $T_{w} \sim 40-55 \mathrm{~K}$ for the warm dust component and $T_{c} \sim 20-23 \mathrm{~K}$ for the cool dust component, both with dust emissivity index $\epsilon=2$. The warm dust, heated by the ionizing stars produced in the starburst, contributes between $40 \%$ and $70 \%$ of the thermal FIR emission (e.g., Chini et al. 1986b; Lonsdale-Persson \& Helou 1987; Young et al. 1989). The rest of the FIR emission is due to the cool dust, which has an associated mass around $10^{7}-10^{8} M_{\odot}$, i.e., between 40 and 150 times the warm dust mass. The resulting gas-to-dust ratio values are compatible, within a factor of $\approx 2$, with the Milky Way 
average (Sodroski et al. 1997) once variations in metallicity are taken into account. The contribution of the cool dust component is, therefore, not negligible even in warm systems such as starburst galaxies, although its importance varies by many factors from galaxy to galaxy. The heating of a fraction of the cool dust component is from the starburst, as the ISRF alone is not sufficient to account for the FIR flux associated with the cool dust. Large dust column densities and nonionizing photons from the ageing stars are the most likely causes for cool dust associated with the starburst (e.g., Lisenfeld et al. 1999).

Around $70 \%$ of the bolometric energy is emitted in the FIR, even if the galaxies in our sample are generally classified as UV bright. Moreover, the UV emission below $0.2 \mu \mathrm{m}$ represents less than $15 \%$ of the FIR emission (with the exception of Tol 1924-416). These estimates are consistent with the amount of stellar light absorbed by dust at UV-tonear-IR wavelengths as predicted by the starburst obscuration curve of Calzetti et al. (1994; Calzetti 1997a). The starburst obscuration curve, applied to SEDs of starforming galaxies with a zero point $R_{V}^{\prime} \simeq 4.05$, reproduces the observed FIR emission within a factor of 2 for individual objects and within $\sim 20 \%$ when averaged over large galaxy samples. The improvement in accuracy is due to the averaging of stellar populations and dust geometry variations in large samples.

The variety of FIR SEDs observed in our sample of local galaxies has direct implications for the detectability of UVbright, high-redshift galaxies at FIR rest-frame wavelengths. If the bolometric luminosities of Lyman-break galaxies are around or above $\sim 3 \times 10^{12} L_{\odot}$ and their FIR SEDs resemble that of NGC 6090, they would be detectable with current submillimeter instrumentation, as the predicted flux at $850 \mu \mathrm{m}$ (observer's frame) is around or greater than $4.4 \mathrm{mJy}$. However, if the dominant FIR SED in highredshift galaxies is like Tol 1924-416, these galaxies will be undetected with the current SCUBA sensitivity limits, no matter how bright the objects intrinsically are. Similar considerations hold for galaxies at $z \sim 1$. There are reasons to believe that dust in $z \sim 3$ galaxies may be hotter than in galaxies at lower redshifts. Star formation is intense and widespread over the entire galaxy in the high-redshift systems (Giavalisco et al. 1996, 2000), thus increasing the number of ionizing photons available per dust grain. In addition, $z \sim 3$ galaxies could be fairly unevolved objects, possibly implying little contribution from aged stellar populations to the dust heating. Both effects would combine to make high-redshift $(z \gtrsim 3)$ galaxies relatively "hot" FIR emitters.

Information on the FIR emission of the Lyman-break galaxies has been recently collected (Chapman et al. 1999), but very little or only partial data on the long-wavelength SEDs of these objects or of the SCUBA sources are currently available (e.g., Lilly et al. 1999a; Smail et al. 1999 and references therein). Measuring dust SEDs is necessary to determine the dust content and emission characteristics of galaxies at high redshift and the relationship between the Lyman-break galaxies and the SCUBA sources. In the lowredshift universe, ISO has only started to unveil the full extent of the parameter space (e.g., range of temperatures, masses, spatial distribution of the different dust components, dependence on metallicity, starburst/host ratio, etc.) of the dust FIR emission in galaxies. Future FIR missions, such as SIRTF, together with submillimeterwavelength observations, will be instrumental for investigating the dust content of local galaxies in statistically meaningful samples.

The authors thank Rosemary Wyse for many useful discussions and comments in different parts and at various stages of this work. D. C. thanks Nino Panagia for discussions on the FIR opacity mechanisms in our Galaxy and for a critical reading of the manuscript. The hospitality of IPAC for two full sessions of data reduction is acknowledged; in particular, D. C. thanks Nanyao Lu for walking her through the mysteries of the data reduction software PIA. Literature searches have made use of the NASA/IPAC Extragalactic Database (NED) which is operated by the Jet Propulsion Laboratory, California Institute of Technology, under contract with the National Aeronautics and Space Administration. This work was partially supported by the NASA ISO grant J-0496/NAG 5-3360.

\section{REFERENCES}

Acosta-Pulido, J. A., et al. 1996, A\&A, 315, L121

Alton, P. B., Trewhella, M., Davies, J. I., Evans, R., Bianchi, S., Gear, W., Thronson, H., Valentijn, E., \& Witt, A. 1998, A\&A, 335, 807

Andreani, P., \& Franceschini, A. 1996, MNRAS, 283, 85

Andriesse, C. D. 1974, A\&A, 37, 257

Barger, A. J., Cowie, L. L., Smail, I., Ivison, R. J., Blain, A. W., \& Kneib, J.-P. 1999, AJ, 117, 2656

Bianchi, S., Davies, J. I., \& Alton, P. B. 1999, A\&A, 344, L1

Blain, A. W., Kneib, J.-P., Ivison, R. J., \& Smail, I. 1999, ApJ, 512, L87

Bohlin, R. C., Savage, B. D., \& Drake, J. F. 1978, ApJ, 224, 132

Boulanger, F., Beichman, C., Désert, F. X., Helou, G., Perault, M., \& Ryter, C. 1988, ApJ, 332, 328

Buat, V., \& Burgarella, D. 1998, A\&A, 334, 772

Calzetti, D. 1997 a, AJ, 113, 162

. 1997b, in AIP Conf. Proc. 408, The Ultraviolet Universe at Low and High Redshift: Probing the Progress of Galaxy Evolution, eds. W. H. Waller, M. N. Fanelli, J. E. Hollis, \& A. C. Danks (Woodbury: AIP), 403

Calzetti, D., Bohlin, R. C., Kinney, A. L., Storchi-Bergmann, T., \&

Heckman, T. M. 1995, ApJ, 443, 136

Calzetti, D., \& Heckman, T. M. 1999, ApJ, 519, 27

Calzetti, D., Kinney, A. L., \& Storchi-Bergmann, T. 1994, ApJ, 429, 582 . 1996, ApJ, 458, 132

Chapman, S. C., et al. 1999, MNRAS, submitted (astro-ph/9909092)

Chini, R., Kreysa, E., Krügel, E., \& Mezger, P. G. 1986a, A\&A, 166, L8

Chini, R., Krügel, E., \& Kreysa, E. 1986b, A\&A, 167, 315

Colbert, J. W., et al. 1999, ApJ, 511, 721

Cox, P., Krügel, E., \& Mezger, P. G. 1986, A\&A, 155, 380
Davies, J. I., Alton, P., Trewhella, M., Evans, R., \& Bianchi, S. 1999, MNRAS, 304, 495

De Mello, D. F., Leitherer, C., \& Heckman, T. M. 2000, ApJ, 530, 251

Désert, F.-X., Boulanger, F., \& Puget, J. L. 1990, A\&A, 237, 215

de Vaucouleurs, G., de Vaucouleurs, A., Corwin, H. G., Jr., Buta, R. J.,

Paturel, G., \& Fouqué, P. 1991, Third Reference Catalogue of Bright Galaxies (New York: Springer)

Dickinson, M. 1998, in The Hubble Deep Field, STScI May Symposium, eds. M. Livio, S. M. Fall, \& P. Madau (Cambridge: Cambridge Univ. Press), 219

Domingue, D. L., Keel, W. C., Ryder, S. D., \& White, R. E. III. 1999, AJ, in press (astro-ph/9906281)

Downes, D. \& Solomon, P. M. 1998, ApJ, 507, 615

Draine, B. T., \& Anderson, N. 1985, ApJ, 292, 494

Draine, B. T., \& Lee, H. M. 1984, ApJ, 307, 431

Eales, S., Lilly, S., Gear, W., Dunne, L., Bond, J. R., Hammer, F., Le Fèvre, O., \& Crampton, D. 1999, ApJ, 515, 518

Giavalisco, M., Steidel, C. C., \& Macchetto, F. M. 1996, ApJ, 470, 189

Giavalisco, M., et al. 2000, in preparation

Gordon, K. A., Calzetti, D., \& Witt, A. N. 1997, ApJ, 487, 625

Gordon, M. A., Heidmann, J., \& Epstein, E. E. 1982, PASP, 94, 415

Goudfrooij, P., \& de Jong, T. 1995, A\&A, 298, 784

Haas, M. 1998, A\&A, 337, Li

Haas, M., Lemke, D., Stickel, M., Hippelein, H., Kunkel, M., Herbstmeier, U., \& Mattila, K. 1998, A\&A, 338, L33

Helou, G. 1986, ApJ, 311, L33

Helou, G., Ryter, C., \& Soifer, B. T. 1991, ApJ, 376, 505

Hildebrand, R. H. 1983, QJRAS, 24, 267 
Hughes, D., et al. 1998, Nature, 394, 241

Kinney, A. L., Bohlin, R. C., Calzetti, D., Panagia, N., \& Wyse, R. F. G. 1993, ApJS, 86, 5

Klaas, U., Haas, M., Heinrichsen, I., \& Schultz, B. 1997, A\&A, 325, L21

Koornneef, J. 1993, ApJ, 403, 581

Krügel, E., Siebenmorgen, R., Zota, V., \& Chini, R. 1998, A\&A, 1998, L9

Kwan, J., \& Xie, S. 1992, ApJ, 398, 105

Leitherer, C., \& Heckman, T. M. 1995, ApJS, 98, 9

Lilly, S. J., Eales, S. A., Gear, W. K. P., Hammer, F., Le Fevre, O., Crampton, D., Bond, J. R., \& Dunne, L. 1999a, ApJ, 518, 641

Lilly, S., Eales, S., Gear, W. K. P., Webb, T., Bond, J., \& Dunne, L. 1999b, in The Formation of Galactic Bulges, Proc. of the workshop held at STScI, October 1998, in preparation (astro-ph/9903157)

Lisenfeld, U., Isaak, K. G., \& Hills, R. 2000, MNRAS, 312, 433

Lonsdale-Persson, C. J., \& Helou, G. 1987, ApJ, 314, 513

Mathis, J. S., Mezger, P. G., \& Panagia, N. 1983, A\&A, 128, 212

Mathis, J. S., \& Whiffen, G. 1989, ApJ, 341, 808

McQuade, K., Calzetti, D., \& Kinney, A. L. 1995, ApJS, 97, 331

Meurer, G. R., Heckman, T. M., \& Calzetti, D. 1999, ApJ, 521, 64

Meurer, G. R., Heckman, T. M., Lehnert, M. D., Leitherer, C., \& Lowenthal, J. 1997, AJ, 114, 54

Mezger, P. G., Mathis, J. S., \& Panagia, N. 1982, A\&A, 105, 372

Natta, A., Palla, F., Panagia, N., \& Preite-Martinez, A. 1981, A\&A, 99, 289

Natta, A., \& Panagia, N. 1976, A\&A, 50, 191

Panagia, N. 1978, in Infrared Astronomy, Proc. Advanced Study Institute, eds. G. Setti \& G. G. Fazio (Dordrecht: Holland), 115

Pei, Y.C., \& Fall, S. M. 1995, ApJ, 454, 69

Pei, Y. C., Fall, S. M., \& Hauser, M. G. 1999, ApJ, 522, 604

Pettini, M., Kellogg, M., Steidel, C. C., Dickinson, M., Adelberger, K. L., \& Giavalisco, M. 1998, ApJ, 508, 539
Pettini, M., Ellison, S., Steidel, C. C., Shapley, A. E., \& Bowen, D. V. 1999, ApJ, in press

Puget, J. L., \& Léger, A. 1989, ARA\&A, 27, 161

Rigopoulou, D., Lawrence, A., \& Rowan-Robinson, M. 1996, MNRAS, 278, 1049

Roche, P. F., \& Chandler, C. J. 1993, MNRAS, 265, 486

Rowan-Robinson, M. 1992, MNRAS, 258, 787

Rowan-Robinson, M., \& Crawford, J. 1989, MNRAS, 238, 523

Rowan-Robinson, M., \& Efstathiou, A. 1993, MNRAS, 263, 675

Sellgren, K. 1984, ApJ, 277, 623

Seki, J., \& Yamamoto, T. 1980, Ap\&SS, 72, 79

Sievers, A. W., Reuter, H.-P., Haslam, C. G. T., Kreysa, E., \& Lemke, R. 1994, A\&A, 281, 681

Smail, I., Ivison, R. J., Owen, F. N., Blain, A. W., \& Kneib, J.-P. 2000, ApJ, 528,612

Sodroski, T. J., Odegard, N., Arendt, R. G., Dwek, E., Weiland, J. L., Hauser, M. G., \& Kelsall, T. 1997, ApJ, 480, 173

Soifer, B., Boehmer, L., Neugebauer, G., \& Sanders, D. 1989, AJ, 98, 766

Steidel, C. C., Adelberger, K. L., Giavalisco, M., Dickinson, M., \& Pettini, M. 1999, ApJ, 519, 1

Steidel, C. C., Giavalisco, M., Pettini, M., Dickinson, M., \& Adelberger, K. L. 1996, ApJ, 462, L17

Storchi-Bergmann, T., Kinney, A. L., \& Challis, P. 1995, ApJS, 98, 103

Storrie-Lombardi, L. J., McMahon, R. G., \& Irwin, M. J. 1996, MNRAS, 283, L79

Wall, W. F. et al. 1996, ApJ, 456, 566

Xu, C., \& Helou, G. 1996, ApJ, 456, 163

Young, J. S., Xie, S., Kenney, J. D. P., \& Rice, W. L. 1989, ApJS, 70, 699

Young, J. S., et al. 1995, ApJS, 98, 219 